\title{
Certainty and Singular Causal Judgment
}

\author{
Kevin G. O'Neill \\ Major Area Paper \\ Spring 2021 \\ Department of Psychology \& Neuroscience \\ Duke University
}

Committee:

Dr. Felipe De Brigard

Dr. John Pearson

Dr. Elika Bergelson

Dr. Benjamin Eva 


\section{Contents}

$\begin{array}{ll}\text { Introduction } & 4\end{array}$

$\begin{array}{ll}\text { Singular Causal Judgment } & 6\end{array}$

Early Counterfactual Accounts . . . . . . . . . . . . . . 6

Advantages of a Lewis-inspired Account. . . . . . . . . . . . . . . . 7

Problems with a Lewis-inspired Account . . . . . . . . . . . . . . . 7

Counterfactual Sampling and Probability . . . . . . . . . . . . . . . . 9

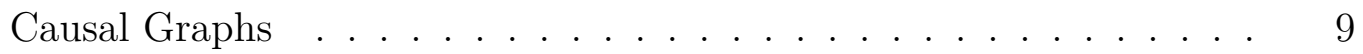

Measures of Singular Causal Strength . . . . . . . . . . . . . . . . . . 13

Why Counterfactual Theories?. . . . . . . . . . . . . . . . . . . . . 17

Alternative Accounts . . . . . . . . . . . . . . . . . . . . . 17

Mental Model Theory . . . . . . . . . . . . . . . . . . . . . . . . 17

Regularity Theories . . . . . . . . . . . . . . . . . . . . . 18

Process Theories of Causal Judgment . . . . . . . . . . . . . . . . . . 18

Social Cognitive Theories . . . . . . . . . . . . . . . . . . . . . . 19

\begin{tabular}{ll}
\hline Metacognition & 19
\end{tabular}

Measuring uncertainty . . . . . . . . . . . . . . . . 20

Self-Report Measures . . . . . . . . . . . . . . . . . . . . . . . . 20

Opt-Out Responses . . . . . . . . . . . . . . . . . . . . 20

Post-Decision Wagers . . . . . . . . . . . . . . . . . 21

Implicit Measures . . . . . . . . . . . . . . . . . . . . . . . . . . . . . 21

Model-based Measures . . . . . . . . . . . . . . . . . . . . . . 21

The Problem with Causal Judgments . . . . . . . . . . . . . . . . . 22

Modeling Uncertainty . . . . . . . . . . . . . . . . . . . . 24

Hypothesis Testing . . . . . . . . . . . . . . . . . . . . . . . . . . . . . . . . . . . . 24

Parameter Estimation . . . . . . . . . . . . . . . 26

$\begin{array}{lr}\text { Confidence in Causal Judgments } & 27\end{array}$

Confidence in General Causal Judgments . . . . . . . . . . . . . . . . . . . . 27

Confidence in Singular Causal Judgments . . . . . . . . . . . . . . . . . . 30

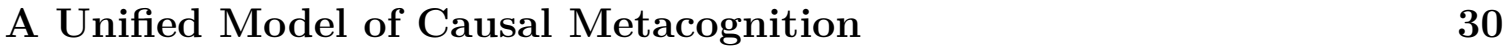

Monte-Carlo Estimation . . . . . . . . . . . . . . . . . . 31

Monte-Carlo Standard Error . . . . . . . . . . . . . . . . . . . . . . . 34

Variance . . . . . . . . . . . . . . . . . . . . . . . 34

Coefficient of Variation . . . . . . . . . . . . . . . . . . . . . . . . 34

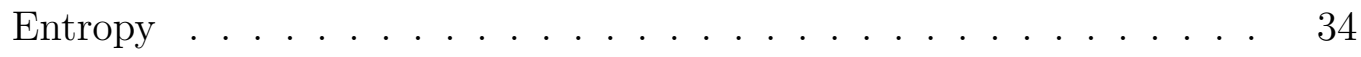

Alternatives to Monte-Carlo Estimation . . . . . . . . . . . . 35

Importance Sampling . . . . . . . . . . . . . . . . . . . . . . . . . . . 35

Causal Structure Induction. . . . . . . . . . . . . . . . . . . . . . . . 37 
\begin{tabular}{ll}
\hline Discussion & 37
\end{tabular} 


\section{Introduction}

Imagine that the mouse on your computer stops working. To fix the problem, you decide to get multiple opinions and call both your brother and a local computer shop. Your brother says that perhaps your computer has a virus preventing the mouse from working, though he isn't entirely sure. The computer shop says that your computer's firmware is out of date, and they guarantee that the out-of date firmware is causing your mouse to malfunction. Given this information, should you first install anti-virus software, or install the firmware update? Intuition suggests that you should probably install the firmware update.

This example highlights that people often qualify causal statements with information about certainty. When your brother says that a virus might have caused your mouse to malfunction, he is not just identifying a potential cause of your broken mouse; he is also making sure to communicate that due to lack of expertise or lack of knowledge, he may be missing something completely different that could have caused this outcome. So if you were to install anti-virus software, then it isn't necessarily the case that your mouse will start working. By contrast, the computer shop expressed their confidence in their solution to communicate that if you were to update your firmware, it is highly likely that your mouse will start working. This certainty also clarifies that manipulating other potential causes (like the computer virus) are unlikely to fix the mouse. In both cases, people qualified causal statements with degrees of certainty to express the perceived likelihood that an intervention on the cause will make a difference for the effect.

Unfortunately, being certain in a causal statement does not always make that statement more accurate. In addition to being certain, we also need to be sure that our increased certainty is reflective of a higher probability of being correct. Take the case of fad diets: suppose a new diet claims that people are healthiest when they minimize consumption of sugar. If a proponent adopts this diet, they are likely to confidently attribute any positive health outcomes (such as weight loss) to the diet as opposed to other factors. But an experienced nutritionist might have less confidence that the diet caused weight loss since many other factors are at play (e.g., perhaps in reducing consumption of sugar, the individual also reduced consumption of processed foods and increased consumption of vegetables). Compared to the expert nutritionist, the proponent of the diet has higher confidence, but this is reflective of overconfidence, not of higher accuracy. This highlights the fact that accuracy and confidence in causal judgments are dissociable; i.e., we can be wrong about causal relationships no matter how certain we are about them, and we can be right about causal relationships no matter how uncertain we are about them.

The ability for causal reasoning is thought to enable people to make sense of their environment and optimize their existence within it. Prospectively, causal selection (i.e., identifying the cause of some event) allows individuals to identify effective ways to produce desired outcome states (Hitchcock \& Knobe, 2009, Morris et al., 2018). In the moment, causal judgment (i.e., weighing the strength of some cause) 
helps people determine who or what is responsible for a state of affairs and decide to praise or blame accordingly (Chockler \& Halpern, 2004 Knobe \& Fraser, 2008; Malle et al., 2014). Retrospectively, people use causal explanations to communicate with others why some particular (un)desired state of affairs exists (Lombrozo, 2007; Lombrozo \& Vasilyeva, 2017; Woodward, 2003). In the field of artificial intelligence, causal reasoning has been identified as one of the major limiting factors of the utility and generalizability of machine learning algorithms, and as a result much work has gone into efficiently learning representations that afford causal conclusions (Dasgupta et al., 2019; Gershman, 2017; Gershman et al., 2015; Pearl, 2019).

But as we saw with the example of the broken computer mouse, the degree to which causal beliefs reflect reality is contingent on our certainty in those beliefs. Furthermore, the fad diet example demonstrated that the accuracy of causal statements also depends on our ability to calibrate our confidence with accuracy to avoid being underconfident or overconfident. Under the assumption that people are better able to intervene on their environment, hold others responsible for the consequences of their actions, and explain the past when their causal models of the world are accurate, causal beliefs are generally more useful when we are confident in them and when this confidence in them is well-calibrated. To understand how people learn to make causal judgments, when causal judgments fail, and how people communicate causal knowledge, then, we need to study certainty. In cognitive psychology and neuroscience, the ability to monitor the accuracy of one's own thought processes is called metacognition. Though the field of metacognition has developed successful models of how people keep track of uncertainty in perception (Maniscalco et al., 2016; Peters \& Lau, 2015; Shekhar \& Rahnev, 2020), memory (Fleming \& Dolan, 2012, Fleming \& Lau, 2014), and decision-making (Adler \& Ma, 2018; Kepecs \& Mainen, 2012; Kiani \& Shadlen, 2009), little is known about how people evaluate uncertainty in causal judgments. Since the usefulness of causal judgments depends on how certain one is about that judgment and the extent to which this certainty is well-calibrated, developing such an account is necessary for an understanding of causal reasoning and causal learning.

In considering certainty in causal judgments, I will distinguish between two kinds of causal relations: general and singular causal relations (Danks, 2017). General causal relations concern whether events of type $C$ cause events of type $E$ in general, including whether smoking causes cancer, whether tax breaks benefit the economy, and whether a fad diet causes weight loss (as in the earlier example). There has been limited research on confidence in judgments of general causal relations (henceforth general causal judgments), though results indicate that models of causal judgment do not account for at least some observed differences in confidence (Liljeholm, 2015; Liljeholm \& Cheng, 2009). In contrast to general causal relations, singular causal relations concern whether this $C$ caused that $E$ in particular, including whether this smoking caused that cancer, whether this tax break benefited that economy, and whether this out-of-date firmware caused that mouse to malfunction (as in the original 
example). Whereas there is some research on confidence in general causal judgments, there has been virtually no research on confidence in singular causal judgments (but see O'Neill et al., 2021). In this paper, I will focus exclusively on singular causal judgments like in the broken mouse example. However, we will see that singular causal judgments depend on knowledge of general causal relations, so it will be critical to study certainty in both of these types of causal relations.

In this paper, I map out broad aims, challenges, predictions, and implications for the resulting intersection of singular causal judgment and metacognition that I (tentatively) call causal metacognition. First, I will overview research on singular causal judgment, focusing on popular counterfactual theories that provide a formal framework for evaluating dependency relationships, as well as several competing definitions of singular causal strength. Next, I will provide relevant background in the literature on metacognition for perception and decision-making, discussing major computational theories of metacognitive judgments. After covering the small amount of work on uncertainty in causal judgments, I will then argue that although singular causal judgments pose a particular problem for some theories of metacognition, counterfactual theories of singular causal judgment already provide testable predictions for confidence in causal judgments and can be extended to account for a wide range of patterns in confidence in singular causal judgments. Finally, I will summarize why we need a study of causal metacognition, and what empirical and theoretical advancements in that field might look like.

\section{Singular Causal Judgment}

Before investigating how people manage and make use of uncertainty in their causal judgments, we first need a basic account of how people identify causes. Although there exist several such accounts, I will focus primarily on counterfactual theories of causal judgment, because these theories have had the greatest success in explaining observed causal judgments in a wide array of experiments, and because they can most readily contribute to a computational theory of causal metacognition (Gerstenberg et al., 2014; Gerstenberg et al., 2020; Gopnik et al., 2004 Griffiths \& Tenenbaum, 2005; Henne, Niemi, et al., 2019; Holyoak \& Cheng, 2011). After reviewing counterfactual theories, I will briefly overview some popular competing theories and argue that they need further extension to explain certainty in causal judgment.

\section{Early Counterfactual Accounts}

A common intuition is that causes are difference-makers. For example, imagine that you flip a switch and a light turns on. We tend to think that flipping the switch caused the light to turn on since, presumably, the light wouldn't have turned on if we didn't flip the switch. The scenario in which we didn't flip the switch is called a counterfactual possibility (i.e., it is counter to the fact that we did flip the switch), and this form of if-then logic using counterfactual possibilities is called 
counterfactual reasoning (Byrne, 2016). In addition to being intuitive notions of dependence, counterfactual definitions of causation are also commonly used in the law (Hart \& Honoré, 1985).

The notion that causes make a difference to their effects can be traced back to Hume, who wrote: "...we may define a cause to be an object, followed by another...where if the first object had not been, the second never had existed" (Hume, 2020). But it was the philosopher David Lewis who formalized this intuition by defining what it means for a counterfactual statement of the form if $C$ happened then $E$ would happen to be true: a counterfactual is true either when it's impossible for $C$ to happen (i.e., it is trivially true), or when some possible world in which both $C$ and $E$ happen more closely resembles the actual world than any world in which $C$ happens but $E$ doesn't. So, then, Lewis took $C$ to cause $E$ if and only if $E$ doesn't happen in the closest possible world in which $C$ doesn't happen (Lewis, 1974). Lewis later expanded this definition to accommodate various philosophical issues, but the general idea that causation reduces to counterfactuals remained (Lewis, 2000).

\section{Advantages of a Lewis-inspired Account}

While Lewis intended this as a definition of what it means for one event to cause another, it is easy to construe this definition as a loose psychological algorithm for modeling how people select causes (regardless of whether those events are in fact causal). I will call this a Lewis-inspired account since it retains the spirit of Lewis (1974) but applies to a domain (i.e., psychology) that Lewis never intended it to work for. The account proceeds as such: when someone wants to determine whether $C$ caused $E$, they imagine the most normal scenario in which $C$ fails to occur, and they simply ask whether $E$ would occur in that world. If $E$ no longer occurs, then one can reason that $C$ made a difference to $E$. So to determine whether the switch caused the light to turn on, one would ask whether the light would turn on if you never flipped the switch. Since it seems most likely that the light would remain off in this scenario, one would conclude that the switch indeed made a difference to the light. In this way, Lewis's simple counterfactual account of causation can be co-opted as a counterfactual account of human causal judgment that captures the deep intuition that causes make a difference to their effects.

\section{Problems with a Lewis-inspired Account}

While this Lewis-inspired account handles a comparatively large array of intuitions and was widely influential for later theories of causal judgment, it is incomplete. Since its philosophical problems have been widely discussed and are less relevant for the purposes of constructing a computational theory of causal metacognition, I will

not discuss them here (see Menzies and Beebee $(2020)$ for an overview of these issues). Instead, I will focus on three explanatory gaps of a Lewis-inspired account as a psychological model of causal judgment: counterfactual distance, causal structure, 
and certainty. Each of these issues can be seen as a consequence of my treating a theory of what causation is as if it were a theory of how people reason causally.

The definition of causation presented by Lewis (1974) depends heavily on a notion of counterfactual distance: whether $C$ makes a difference to $E$ depends on what would happen in the absence of $C$ and how similar this possible world is to the actual world. To Lewis, counterfactual distance is the extent to which the possible world resembles the actual world, or how many facts about the actual world need to be altered to obtain the possible world. Lewis later provided a more sophisticated definition of counterfactual distance, but also acknowledged that this definition may be context-sensitive (Lewis, 1979, 2000). For Lewis's theory of what causation is, it does not matter whether or how counterfactual distance is computed: all that matters is that counterfactual distance exists and that it helps define causation. But a psychological account must fill this gap. In particular, the Lewis-inspired account needs a process by which people come to determine which counterfactual possibility is closest to the real world that explains precisely which counterfactual possibilities one considers and how exactly they are compared to each other. Since there are an infinite number of such possibilities and an infinite number of facts to consider about each possibility, such an account has eluded explication. Given how much Lewis's account of causation relies on counterfactual distance, we can only use this account to describe human behavior insofar as we can pin down what counterfactual distance amounts to and how it can be computed in a psychologically tractable manner.

Likewise, it does not concern Lewis (1974) how one can know whether $E$ would happen if $C$ did not: all that matters is that there is some fact of the matter as to whether $E$ would happen in the closest possible world in which $C$ did not happen. We call this set of counterfactual dependence relations the causal structure, since it is the overarching structure that determines which counterfactual worlds are possible and which are impossible. But again, if we are interested in how people judge causes, then we need to specify how people can learn and reason with causal structures. Without being able to determine whether a participant thinks $E$ would occur in the absence of $C$, it is impossible to determine whether the participant thinks $C$ caused $E$.

Most importantly for the purposes of this paper, the Lewis-inspired account provides no basis upon which to determine how certain one is that $C$ caused $E$. Since only one counterfactual possibility is relevant for determining whether $C$ caused $E$ (i.e., the one closest to the actual world in which $C$ does not occur), an inferred causal relation simply exists or it does not. So long as one found the closest counterfactual possibility, one should be always fully confident in whatever judgment one made. This is a desirable property for Lewis (1974), since there should be a fact of the matter for any given causal claim. But since people appear to have uncertainty and be able to assess their uncertainty in causal claims, a psychological theory of causal judgment should account for that uncertainty (Collins \& Shanks, 2006 Liljeholm, 2015. Liljeholm \& Cheng, 2009; O'Neill et al., 2021; Perales \& Shanks, 2003; Shanks, 1987). 


\section{Counterfactual Sampling and Probability}

It should not be terribly surprising that the Lewis-inspired account does not suffice as a psychological account of certainty in causal judgment; after all, Lewis (1974) sought to describe what causation is, not how we reason causally. But recent extensions of the general idea that counterfactuals underlie causal judgment have gained much popularity for their ability to explain how causal judgments are sensitive to dependence between events (Cheng \& Novick, 1990 Davis \& Rehder, 2020 Pearl, 2009), normality (Gerstenberg \& Icard, 2020; Henne et al., 2021; Hitchcock \& Knobe, 2009 Icard et al., 2017; Kirfel \& Lagnado, 2019; Knobe \& Fraser, 2008; Kominsky \& Phillips, 2019: Sytsma, 2019), whether the events are actions or omissions (Henne, Bello, et al., 2019; Henne, Niemi, et al., 2019, Henne et al., 2017), the presence of alternative causes (Kominsky et al., 2015; Lu et al., 2008; Morris et al., 2019; O'Neill et al., 2021), and the perceived effectiveness of an intervention on the cause (Kushnir \& Gopnik, 2005 Lagnado \& Sloman, 2004; Morris et al., 2018; Sobel \& Kushnir, 2006; Woodward, 2003) among other factors.

Counterfactual sampling models extend the proposal by Lewis (1974) in three important ways. First, these models make use of a formalism called causal graphs that express relationships between variables to constrain the space of counterfactuals to those that are internally consistent (Pearl, 2009). Second, these models assume that people consider not just one, but a wide range of alternative possibilities in determining whether $C$ caused $E$. Third, they assume that people probabilistically estimate the extent to which $C$ caused $E$ by averaging a measure of causal strength over all of the considered possibilities. Together, these advances transform Lewis's metaphysics of causation into a class of computational cognitive models for causal judgment. Each of these models consists of two components: a causal graph that is common across models, and a measure of causal strength that varies between models. I will now describe each of these components in turn.

\section{Causal Graphs}

A causal graph $\mathcal{G}=(\mathcal{U}, \mathcal{V}, \mathcal{F})$ (also known as a structural causal model or causal Bayesian network) is a mathematical formalism used to define the functional relationships between a set of variables, and consequently, the space of possible value assignments to each of those variables (Hall, 2007; Pearl, 2009). It is the combination of a set of exogenous variables $\mathcal{U}$ with unspecified causes (often independent error terms), a set of endogenous variables $\mathcal{V}$ that are fully determined by the other endogenous and exogenous variables, and a set of functional relations $\mathcal{F}$ which define for each endogenous variable $v \in \mathcal{V}$ a functional relation $f \in \mathcal{F}$ that assigns $v$ in terms of the other variables $\mathcal{U} \cup \mathcal{V} \backslash\{v\}$ (where $\backslash$ is the set difference and $\mathcal{U} \cup \mathcal{V} \backslash\{v\}$ is the set of all variables besides $v$ ). We call this a causal graph because if you represent variables as nodes and functional relations as edges, you end up with a graph that neatly summarizes the influences of the variables on each other. For instance, if we 
wanted to create a causal graph that encoded the relationships between the state of the light switch $(S \in\{0,1\})$ and whether or not the light is lit $(L \in\{0,1\})$, we could make the causal graph $\mathcal{U}=\{S\}, \mathcal{V}=\{L\}, \mathcal{F}=\{L=S\}$, represented in Figure 1 .

\section{Figure 1}

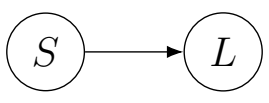

Causal graph of the relationship between a switch (S) and a light (L). In this graph, the value of $L$ is determined solely by the value of $S$.

There has been a large amount of work on how to infer the structure of a graph given a joint probability distribution over its variables (Griffiths \& Tenenbaum, 2005: Meder et al., 2014; Pearl, 2009, Spirtes, 2010; Tenenbaum \& Griffiths, 2001). Though causal graph inference arguably constitutes most of the problem of causal judgment, this problem has been shown to be computationally intractable for graphs involving even a modest number of variables (Chickering, 1996; Chickering et al., 2004). For the remainder of the paper, then, I will (in line with previous work on singular causal judgment) assume that the relevant causal graph is fully specified, and that participants making causal judgments have an accurate knowledge of this graph (Halpern \& Hitchcock, 2015; Icard et al., 2017; Morris et al., 2018; Quillien, 2020). To make predictions with a causal graph, one needs the values of all exogenous variables. Then, the values of each endogenous variable are computed using the functions in $\mathcal{F}$ in a sequence such that all of a variable's inputs are computed before the variable itself is computed. Because of this ordering constraint, causal graphs are usually restricted to be acyclic, meaning there is at least one partial ordering $<$ of variables such that for each pair of variables $X$ and $Y$ where $X<Y, X$ is independent of $Y$. For the graph in Figure 1, it means that the value of $S$ is determined independently of $L$. If we assign $S=1$, we can compute $L=S=1$ using the equation in $\mathcal{F}$, resulting in a fully specified possibility $\{S=1, L=1\}$. Likewise, if we assign $S=0$, we can compute $L=S=0$, specifying the possibility $\{S=0, L=0\}$. Since these two possibilities exhaust the domain of $S$, they are the only two possibilities afforded by this causal graph.

For the purposes of this paper, there are three other relevant operations one can perform with causal graphs: conditioning on variables, intervening on variables, and computing counterfactuals. To distinguish these operations, I will use a slightly more complicated scenario. Imagine now that the switch $S \in\{0,1\}$ turns on two different light bulbs $L_{1}, L_{2} \in\{0,1\}$. To account for noise, we can include corresponding independent error terms $U_{S}, U_{L 1}$, and $U_{L 2}$. If we assume the functional relationships $\mathcal{F}=\left\{S=U_{S}, L_{1}=S+U_{L 1}, L_{2}=S+U_{L 2}\right\}$, we end up with the graph depicted in Figure 2 (as discussed in Pearl et al., 2016). Clearly, $L_{1}$ and $L_{2}$ are dependent on $S$, because there are links between them in our graph. 


\section{Figure 2}

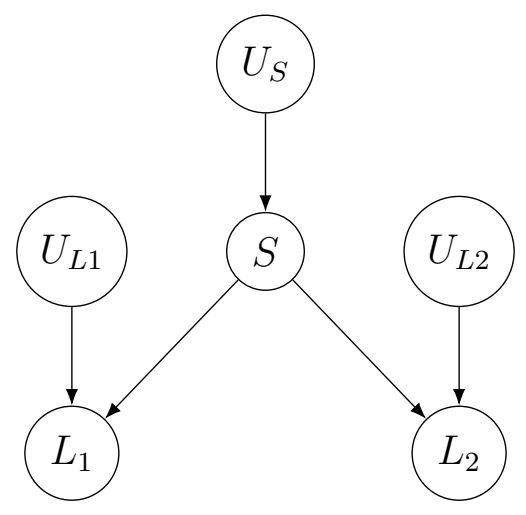

Causal graph of the relationship between a switch ( $S$ ) and two lights $\left(L_{1}\right.$ and $\left.L_{2}\right)$. In this graph, the values of $L_{1}$ and $L_{2}$ are determined both by the value of $S$ and also the value of the error terms $U_{L 1}$ and $U_{L 2}$.

Conditioning. To condition on a value $x$ of a variable $X$ is to identify a subset of the data in which $X=x$, and to estimate some quantity over that subset of the data. Conditioning is good for identifying associations between variables, but does not necessarily tell us about causation. For example, it might be tempting to conclude that $L_{1}$ causes $L_{2}$ if we only use conditioning. If we condition our graph in Figure 2 on $L_{1}=1$, then we will be looking at the subset of our data in which $L_{1}=1$. Here it is likely that $L_{2}=1$ (i.e., $P\left(L_{2}=1 \mid L_{1}=1\right)$ is high), because presumably the reason that $L_{1}=1$ is that $S=1$. If we condition on $L_{1}=0$, then we get the opposite result: $P\left(L_{2}=1 \mid L_{1}=0\right)$ is low since it is likely that $S=0$. If we take the difference of these probabilities (equivalent to the regression coefficient between $L_{1}$ and $L_{2}$ ), we would be tempted to conclude that $L_{1}$ causes $L_{2}$. This is the paradigmatic case of confounding, where the relation between $L_{1}$ and $L_{2}$ is completely explained by $S$.

Intervening. To get around the problem of confounding, we need another operation on our causal graph. One way is to see what would happen in a controlled experiment in which we vary $L_{1}$ and compute the expected values of $L_{2}$. In general this experiment on $L_{1}$ need not be physically possible, as long as it is coherent to fix the value of $L_{1}$ without altering the other variables. The way we do this with causal graphs is to sever all incoming edges to $L_{1}$, and fix the assignment of $L_{1}$ to $L_{1}=1$ or $L_{1}=0$, resulting in the graph depicted in Figure 3 . By fixing the value of $L_{1}=0$ (for instance by untwisting the light bulb), we remove the dependence of $L_{1}$ on $S$ and $U_{L 1}$. So we would see that $L_{2}$ turns on and off with $S$, but $L_{1}$ remains off. So we could infer that $P\left(L_{2}=1 \mid d o\left(L_{1}=0\right)\right)=P(S=1)$, where $d o\left(L_{1}=0\right)$ indicates our intervention on $L_{1}$. Likewise, if we fixed $L_{1}=1$ (for instance by wiring $L_{1}$ to a battery, we would again see that $L_{2}$ co-varies with $S$, and so $P\left(L_{2}=1 \mid d o\left(L_{1}=1\right)\right)=P(S=1)$. Taking the difference of these two probabilities yields an average causal effect (ACE) of 0 , which is consistent with the intuition that common effects of a cause do not cause 
each other.

\section{Figure 3}

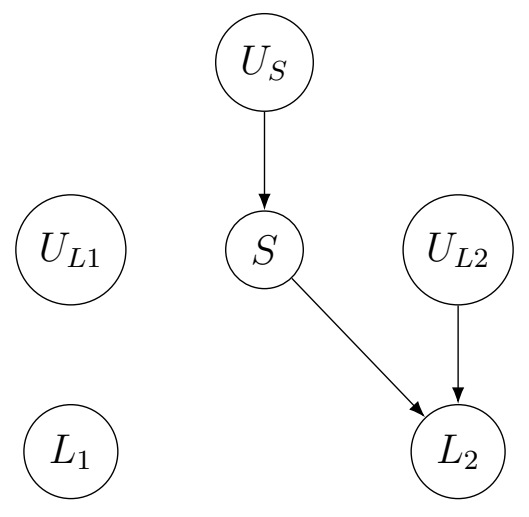

Causal graph of the relationship between a switch $(S)$ and two lights $\left(L_{1}\right.$ and $\left.L_{2}\right)$, where we intervene to fix the value of $L_{1}$. In this graph, $L_{2}$ is determined by both $S$ and $U_{L 2}$, but the value of $L_{1}$ no longer depends $S$ or $U_{L 2}$.

Though intervention is a plausible mechanism for general causal judgments (e.g., that flipping the switch causes the light to turn on in general, it does not suffice as a model of singular causal judgment. To see why, imagine that we're investigating the relationship between smoking and lung cancer, and we find that on average, smoking causes lung cancer (i.e., smoking has a significant ACE on cancer). One of our study participants, however, reported having lung cancer without smoking. Did this participant's smoking cause their lung cancer? Clearly not, because they never smoked. To match our intuitions of singular causal judgments, then, we will need yet another mechanism.

Counterfactual. To recover a useful definition of counterfactuals in causal graphs, we can return to the definition of counterfactuals presented above: a counterfactual is a statement of the form "if $C=c$ were the case, then $E=e$ would be the case." Thankfully, this statement almost exactly matches the definition of the expected value of an intervention $\mathbb{E}[E \mid d o(C=c)]=e$ : "if we fix $C=c$ over the population, we can expect that $E=e$ on average." Here $\mathbb{E}[X]$ is the expected value (or average) of a random variable $X, \mathbb{E}[X \mid Y]$ is the conditional expected value of $X$ given the value of another variable $Y$, and so $\mathbb{E}[E \mid d o(C=c)]$ is the expected value of $E$ given an intervention that fixes the variable $C$ to a value $c$. The only caveat is that we are interested in intervening on particular events, not distributions of events. Since the do operator is defined over probability distributions, we will invoke yet another notation: let us represent a counterfactual of the form "if $C=c$ were the case in situation $U=u$, then $E=e$ would be the case" as $E_{C=c}(u)=e$ (Pearl et al., 2016).

To compute this quantity, we need to perform three steps. First, we need to infer the values of the unobserved exogenous variables using abduction over the values over the observed variables. For deterministic causal graphs including the examples 
above, this is as simple as solving for their values. In the probabilistic case, we must estimate a probability distribution $P(U)$. Next, we modify the graph according to the intervention and fix our values of interest. Finally, we can use the modified graph and the inferred values of $U$ to predict the value of $E$ for that counterfactual.

Suppose that for the two-light scenario in Figure 2 we flip the switch off so that both lights are also off. Would $L_{1}=1$ if we hadn't flipped the switch (i.e., does $\left.L_{1_{S=s}}(u)=1\right)$ ? To find out, we can first infer that since $L_{1}=L_{2}=S=0$, then it must be true that $U_{L 1}=U_{L 2}=U_{S}=0$. Then, we modify the graph to remove all incoming connections to $S$ (not shown) and fix $S=1$. Finally, we can compute that $L_{1_{S=s}}(u)=1$. Though the result itself isn't very surprising in this case, the ability for causal graph to compute such counterfactuals means that they are an ideal candidate for a model of singular causal judgment.

\section{Measures of Singular Causal Strength}

Now that we have a suitable formalism for computing counterfactuals, the only thing left is to develop a measure of singular causal strength in terms of counterfactuals that aligns with observed causal judgments. However, this last step has proven to be exceedingly difficult, and many counterfactual theories diverge at this point. There are too many measures to cover them all, and some argue that a single measure of causal strength is not sufficient to explain singular causal judgments in general (Godfrey-Smith, 2009; Hitchcock, 2007; Lombrozo, 2010, Sprenger, 2018). As such, I will cover a selection of notable measures (for some other notable measures, see Kaiserman, 2018). I will not commit to any particular measure for the purposes of this paper, rather I will commit broadly to the general idea that causal judgments are made by sampling counterfactuals and computing some measure of causal strength across the sampled counterfactuals. For simplicity, I will assume that all variables are binary, and will use the condensed notations $P(E)$ and $P(\neg E)$ to refer to $P(E=1)$ and $P(E=0)$, respectively. Each of the following measures of causal strength specifies a Monte Carlo sampling algorithm that approximates the expected change of the effect $E$ with respect to one or more interventions on the candidate cause $C$. This algorithm can be seen as an instance of Bayesian inference using prior probabilities $P(\mathcal{U})$ over the exogenous variables and a single observation $\mathcal{U}=u, \mathcal{V}=v$ to estimate the expected change in $E$ across a large set of counterfactual possibilities where the value of $C$ is manipulated. I will denote the influence of $C$ on $E$ within a given counterfactual possibility as $\kappa_{n, C \rightarrow E}$, and the aggregate measure of the causal influence of $C$ on $E$ across all possibilities as $\kappa_{C \rightarrow E}$. I follow Icard et al. (2017) in assuming that people's prior probability of sampling a given counterfactual is in proportion to its statistical probability and prescriptive normality. That is, people are more likely to imagine a normal alternative to an abnormal outcome than they are to imagine an abnormal alternative to a normal outcome (I will relax this assumption in the section Importance Sampling; Kahneman \& Tversky, 1981). Thus, each measure $\kappa_{C \rightarrow E}$ can be specified in an iterative algorithm (where $n \in\{1 \ldots N\}$ is the iteration number) of 
the form:

1. Sample a set of possible worlds by sampling all exogenous variables $U_{n}$ according to their prior probabilities $P\left(U_{n}\right)$

2. Determine whether the cause $\left(C_{n}\right)$ and effect $\left(E_{n}\right)$ occur in each world by calculating the values of all endogenous variables using the sampled values of $U_{n}$ and the structural equations provided by $\mathcal{F}$

3. Determine the specific causal effect $\kappa_{n, C \rightarrow E}$ in each world as a function of $E_{n}$ and some computed counterfactual(s) $E_{n_{C_{n}=c}}\left(U_{n}\right)$

4. Average all of the specific causal effects to obtain $\kappa_{C \rightarrow E}=\frac{1}{N} \sum \kappa_{n, C \rightarrow E}$

To avoid repeating this algorithm for each measure, I will provide definitions for both the averaged causal strength measure $\kappa_{C \rightarrow E}$ and the causal strength measures specific to each sampled possibility $\kappa_{n, C \rightarrow E}$. Though I provide formal definitions of each measure for completeness, my proposed extensions of counterfactual sampling models do not depend on the details of any particular measure (though I will use Quillien (2020) as an example). Since the definitions of the models are rather technical, some readers may prefer to skip to the next section Why Counterfactual Theories?

Crediting Causality. The Crediting Causality $(C C)$ model predicts that people think that $C$ is a cause of $E$ to the extent that $C$ raises the perceived probability of $E$ relative to the marginal probability of $E$ : that is, $C C_{C \rightarrow E}=P(E \mid C)-P(E)$ (Spellman, 1997). While this measure was intended as a measure for general causal judgments, it can be construed as a measure for singular causal judgments by replacing conditional probabilities with probabilities over a set of counterfactuals:

$$
C C_{C \rightarrow E}=P\left(E_{C=1}\right)-P(E)
$$

Again, $C C$ measures how much more likely $E$ would be to occur if $C$ were always true. To estimate $C C_{C \rightarrow E}$ using Monte-Carlo sampling, one would average the specific causal effects $C C_{n, C \rightarrow E}=E_{n_{C_{n}=1}}\left(U_{n}\right)-E_{n}$, which represent whether intervening to make $C$ happen made a difference to $E$ in a particular world. Note that $C C_{n, C \rightarrow E}$ is only non-zero when $C=0$ : when the cause is present in a sampled world, intervening to set $C=1$ has no effect on $E$ since $C$ is already present. Overall, the CC model predicts that people judge $C$ to cause $E$ when it makes $E$ more likely.

Delta-P. Similar to $C C$, the Delta-P $(\Delta P)$ model predicts that people think that $C$ caused $E$ to the extent that $C$ raises the probability of $E$ relative to its probability when $C$ doesn't occur: $\Delta P_{C \rightarrow E}=P(E \mid C)-P(E \mid \neg C)$ (Jenkins \& Ward, 1965 Rescorla, 1968; Shanks, 1987). This is the same as $C C$, with the modification that whereas $C C$ requires that $C$ raises the overall likelihood of $E, \Delta P$ constrains this to require that $E$ is more likely when $C$ is present than when it is absent. Since $\Delta P$ was also originally formulated as a measure of general causal strength, we can 
similarly turn it into a measure of singular causal strength by changing conditions with counterfactuals:

$$
\Delta P_{C \rightarrow E}=P\left(E_{C=1}\right)-P\left(E_{C=0}\right)
$$

Also known as the average causal effect (ACE), the regression coefficient, or the covariation between $C$ and $E$, this measures the difference in $E$ when $C$ is manipulated to be present compared to when it is manipulated to be absent. Finally, to estimate $\Delta P_{C \rightarrow E}$ with Monte-Carlo sampling, we can average the specific causal effects $\Delta P_{n, C \rightarrow E}=E_{n_{C_{n}=1}}\left(U_{n}\right)-E_{n_{C_{n}=0}}\left(U_{n}\right)$, which specify whether $E$ is different when $C$ is present/absent in a particular possible world.

Power PC. The Power PC $(P P C)$ model is an extension of $\triangle P$ that qualifies when covariation (as measured by $\Delta P$ ) is sufficient to infer causation (Cheng, 1997). Specifically, PPC normalizes $\Delta P$ by the probability that $E$ fails to occur when $C$ doesn't occur: $P P C_{C \rightarrow E}=\frac{\Delta P}{P(\neg E \mid \neg C)}=\frac{P(E \mid C)-P(E \mid \neg C)}{P(\neg E \mid \neg C)}$. When no alternative causes are present, $E$ is unlikely to occur in the absence of $C$ (i.e., $P(\neg E \mid \neg C) \approx 1$ ), and so the extent to which $C$ causes $E$ is just the covariation between $C$ and $E$ (i.e., $\Delta P$ ). But when alternative causes are present, $E$ is likely to occur in the absence of $C$ (i.e., $P(\neg E \mid \neg C) \approx 0$ ), and so $P P C$ becomes undefined. In this case, covariation does not equal causation since $E$ almost always occurs regardless of $C$. To make PPC as a measure of singular causal strength, we can once again change conditions with counterfactuals:

$$
P P C_{C \rightarrow E}=\frac{P\left(E_{C=1}\right)-P\left(E_{C=0}\right)}{P\left(\neg E_{C=0}\right)}
$$

Finally, to estimate $P P C_{C \rightarrow E}$ with Monte-Carlo sampling, we can average

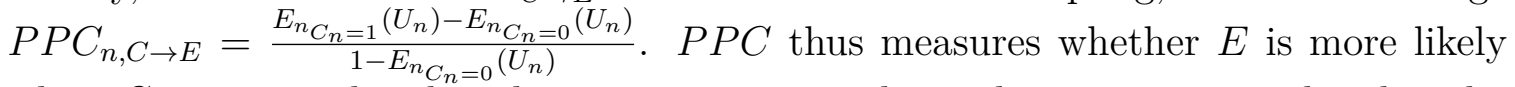
when $C$ is manipulated to be present compared to when it is manipulated to be absent, given that $E$ does not always occur in the absence of $C$.

Probability of Necessity and Sufficiency. Along with his groundbreaking formulation of causal graphs, Pearl proposed three related measures of singular causal strength (Pearl, 2009). Probability of necessity (PN) is the degree to which $C$ is required for $E$, or the probability that $E$ wouldn't have happened if $C$ didn't happen, $P N_{C \rightarrow E}=P\left(\neg E_{C=0} \mid C, E\right)$. Likewise, the probability of sufficiency (PS) is the degree to which $C$ is enough for $E$, or the probability that $E$ would have happened if $C$ happened, $P S_{C \rightarrow E}=P\left(E_{C=1} \mid \neg C, \neg E\right)$. Finally, the probability of necessity and sufficiency (PNS) is the probability that $C$ was both necessary and sufficient for $E$ :

$$
\begin{aligned}
P N S_{C \rightarrow E} & =P\left(\neg E_{C=0}, E_{C=1}\right) \\
& =P(C, E) P N_{C \rightarrow E}+P(\neg C, \neg E) P S_{C \rightarrow E} \\
& =P(C, E) P\left(\neg E_{C=0} \mid C, E\right)+P(\neg C, \neg E) P\left(E_{C=1} \mid \neg C, \neg E\right)
\end{aligned}
$$

So, the three measures capture the degree to which $C$ is required for $E$ to occur, the degree to which $C$ is enough for $E$ to occur, and the degree to which $C$ is both required 
and enough for $E$ to occur. To estimate these measures with Monte-Carlo sampling, we can average the values $P N_{n_{C \rightarrow E}}=1-E_{n_{C_{n}=0}}\left(U_{n}\right)$ in samples where $C_{n}=E_{n}=1$, average the values $P S_{n_{C \rightarrow E}}=E_{n_{C_{n}=1}}\left(U_{n}\right)$ in samples where $C_{n}=E_{n}=0$, and compute the weighted average of the values $P N S_{n_{C \rightarrow E}}=C_{n} E_{n}\left(1-E_{n_{C_{n}=0}}\left(U_{n}\right)\right)+$ $\left(1-C_{n}\right)\left(1-E_{n}\right) E_{n_{C_{n}=1}}\left(U_{n}\right)$. Collectively, these measures are sensitive to whether removing $C$ removes $E$, whether forcing $C$ forces $E$, or both.

Necessity-Sufficiency. Similar to PNS, Icard et al. (2017) presented a measure of causal strength that is a weighted average of how necessary and sufficient (NS) the cause is for the effect. They defined the extent to which $C$ to caused $E$ as the weighted average of the probability that $E$ can't occur without $C$ and the probability that $E$ occurs in the presence of $C$ :

$$
N S_{C \rightarrow E}=P(\neg C) P\left(\neg E_{C=0}\right)+P(C) P\left(E_{C=1}\right)
$$

To estimate $N S$ with Monte-Carlo sampling, we can average the values $N S_{n, C \rightarrow E}=$ $\left(1-C_{n}\right)\left(1-E_{C=0}\right)+C_{n} E_{C=1}$. Like $P N S$, this measure is sensitive to whether removing $C$ removes $E$ and whether forcing $C$ forces $E$. Among other effects, this model explains that singular causal judgments are sensitive to the normality of the cause and the causal structure (Morris et al., 2019). The difference between the two models is that whereas PNS assumes that necessity is only relevant when both $C$ and $E$ occur (since stopping $C$ could've stopped $E$ ) and sufficiency is only relevant when neither $C$ nor $E$ occurs (since forcing $C$ could've forced $E$ ), $N S$ assumes that the relevance of necessity and sufficiency does not depend on whether $E$ happens.

Quillien. Aiming to improve model fit to experimental observations of singular causal judgments, Quillien (2020) proposed a new model based on the intuition that causes are portable; that is, causes make a difference to their effects in a wide array of circumstances (Hitchcock, 2012 Lombrozo, 2007). Essentially, this measure (which I call $Q$ ) is the standardized average causal effect of $C$ on $E$ across the sampled possibilities:

$$
Q_{C \rightarrow E}=\mathbb{E}\left[\frac{E_{n_{C=C_{n}^{\prime}}}\left(U_{n}\right)-E_{n}}{C_{n}^{\prime}-C_{n}}\right] \frac{\sigma_{C}}{\sigma_{E}}
$$

where $C_{n}^{\prime} \neq C_{n}$ is a new randomly sampled value of $C_{n}$, or simply $1-C_{n}$ in the binary case. Intuitively, this is just the expected unit change in $E$ relative to a unit increase in $C$. When the no-confounding assumption holds (i.e., when $C$ has a general causal influence on $E, E$ has no causal influence on $C$, and there is no variable that causes both $C$ and $E$ ), this measure of causal strength reduces to the correlation coefficient between $C$ and $E$ across the imagined possibilities. Compared to observed causal judgments to two different two-cause structures, $Q$ provided a better fit to the data than any of the above models (Morris et al., 2019). 


\section{Why Counterfactual Theories?}

The framework of causal graphs provided a mathematical formalism for defining counterfactuals, explaining the deep connections between intervention and causation, and deriving measures of singular causal strength that yield quantitative predictions of singular causal judgment. While these are certainly impressive feats, there is one quality of counterfactual sampling accounts of causal judgment that makes them ideally suited for explaining certainty in causal judgment: they express causal statements in terms of estimated probabilities. As we will see in the next section on meta-cognition, this formulation is compatible with leading models of certainty judgments in decision-making tasks that also rely on probability distributions to express uncertainty.

\section{Alternative Accounts}

Probabilistic counterfactual sampling accounts of causal judgment are not the only accounts. To defend my focus on this class of theories, I will consider three popular alternative accounts, and argue that each of these accounts need further work before they can contribute to a computational account of confidence in singular causal judgment. Note that while I do argue that these models (as currently formulated) cannot explain certainty in causal judgment, I do not argue that these models are worse than counterfactual models in explaining causal judgments themselves, or that they cannot be modified to explain certainty in causal judgment. Indeed, if future experiments on certainty in causal judgment demonstrate evidence against the normative predictions of counterfactual sampling models that I develop in the section $A$ Unified Model of Causal Metacognition, there very well may be reason to revoke counterfactual models as models of causal judgment. But absent such evidence, there is no reason to augment the following alternative accounts with explanations of uncertainty when counterfactual accounts already provide a basis for such an explanation.

\section{Mental Model Theory}

Not all counterfactual accounts of causal judgment assume that people estimate counterfactual dependence probabilistically. Notably, the causal mental model theory is a dual-process counterfactual theory that assumes that people imagine a very small set of counterfactual possibilities, and match the set of possibilities against templates for different causal relations (e.g., causation, prevention, enabling; Goldvarg \& Johnson-Laird, 2001; Johnson-Laird \& Khemlani, 2017; Khemlani et al., 2014). This basic model has been extended to explain errors in causal inferences, judgments of omissive causes (e.g., not watering plants caused them to die), and even eye movements during online causal judgment (Bello et al., 2018, Henne, Bello, et al., 2019; Khemlani et al., 2018). Though the mental models theory remains a viable account of causal judgment, it does not have an existing theoretical mechanism to account for certainty in causal judgment. Such predictions could be made by, for instance, 
weighting sampled possibilities according to perceived likelihood and counterfactual similarity, or by relating certainty to the number of sampled possibilities (De Brigard et al., 2021). But without theoretical justification and implementation of such extensions, the mental model theory cannot explain certainty in causal judgments.

\section{Regularity Theories}

Regularity theories are similar to counterfactual theories in that they invoke notions of dependence to explain causal judgments, but instead of using the framework of causal graphs, they use the framework of propositional logic. For example, Mackie held that $C$ is a cause of $C$ when $C$ must be an insufficient but necessary part of an unnecessary but sufficient set of conditions (called an INUS condition) for $E$ (Mackie, 1965). A related definition is that $C$ caused $E$ if $C$ is a necessary element of a sufficient set of conditions (called a NESS condition) for $E$ (Wright, 1987). While these sorts of definitions have been influential in how causation is defined and used in the law (and seem on the surface to be equivalent to counterfactual accounts), regularity accounts suffer from the unidirectionality of causal relations (Paul et al., 2013; Pearl, 2009). Considering the causal relationship "disease $D$ causes symptoms $S$ ", for instance, intuition suggests that alleviating the symptoms would have no bearing on whether or not one has the disease. But the material conditional $D \rightarrow S$ (read as "if one has the disease, then one has the symptoms") is logically equivalent with its contrapositive $\neg S \rightarrow \neg D$ (read as "if one doesn't have the symptoms, one doesn't have the disease"). Since logical conditionals can be inverted in this way, regularity accounts fail to account for the fact that causal relations are unidirectional (e.g., that treating one's symptoms does not cure the disease). Furthermore, regularity accounts do not have an available mechanism for explaining certainty in causal judgment. Any given candidate cause $C$ is either an INUS/NESS condition for $E$, or it is not. On the basis of deduction, then, one should always be fully confident that $C$ is or is not a cause of $E$, given that a proof that $C$ is an INUS/NESS condition of $E$ exists. Without expanding regularity accounts with an account of how one can be more or less certain that $C$ is an INUS/NESS condition of $E$, then, such accounts cannot readily inform a study of certainty in causal judgments.

\section{Process Theories of Causal Judgment}

Another common intuition is that causes produce their effects through some physical process (Dowe, 2009; Salmon, 1994). Process theories of causation and causal judgment follow this intuition in stating that $C$ caused $E$ when $C$ transfers some conserved quantity (e.g., force, momentum, energy) to $E$. The force dynamic theory assumes that people also judge causes in this way, and is able to account for causal judgments of different sorts (e.g., causation, prevention, helping, enabling) in various physical scenarios (Wolff, 2007; Wolff et al., 2010). In relation to grounding a study of certainty in causal judgment, however, the force dynamic theory has two major disadvantages. First, since process theories focus on exchanges of quantities, 
they all but fail to explain judgments of omissive causes (e.g., the lack of a firmware update caused the computer mouse to stop functioning). Process theories, then, do not readily account for the fact that people can consistently report confidence for omissive causal judgments (Bernstein, 2014; O'Neill et al., 2021). The second problem is that process theories do not have an established mechanism for explaining certainty in causal judgment. Though Wolff (2007) argues that the force dynamic theory allows for people to be more or less certain in a causal judgment given that they are more or less certain in the magnitudes and direction of relevant forces, he does not provide an account that makes any qualitative or quantitative predictions. Absent such an account, then, process theories will not suffice to guide a study of certainty in causal judgment.

\section{Social Cognitive Theories}

Yet another class of theories, including the culpable control model, suggests that people determine $C$ to be a cause of $E$ when they have a negative evaluation of $C$ and they want to confirm previous ascriptions of blame to $C$ for $E$ (Alicke et al., 2011). The culpable control model is able to explain, among other effects, that people view a norm-violating agent as a greater cause of an effect than a norm-compliant agent when the effect is negative, but the reverse is true when the effect is positive (Alicke \& Rose, 2012; Alicke et al., 2011). However, this model does not predict causal judgments of inanimate objects without appealing to anthropomorphism of simple objects (Henne et al., 2021; Kominsky \& Phillips, 2019). More importantly, the culpable control model does not readily provide any explanation of certainty in causal judgment. Though Alicke et al. (2011) demonstrate that the extent to which ascriptions of blame predict causal judgments is modulated by ambiguity in causal relations, they do not provide an account of where this ambiguity in causal relations comes from. As with the previous models, the culpable control model needs to be augmented with such an account before it can prove useful as a model of certainty in causal judgment.

\section{Metacognition}

I have argued that counterfactual sampling models of causal judgment provide an ideal basis upon which to form an account of certainty in singular causal judgments. The next step towards developing such an account is to identify measures and models of metacognitive uncertainty that are compatible with counterfactual sampling accounts. In this section I will review broad classes of models of metacognitive uncertainty that have been applied to perceptual, decision-making, and memory tasks. My goal here is not to evaluate the evidence for and against each model within the domains that created them, but rather to select the model that most readily generalizes to certainty in singular causal judgments. 


\section{Measuring uncertainty}

Before reviewing models of metacognition, it is worth briefly discussing how researchers in metacognition determine how certain their participants are in a given response, since it is precisely responses on these measures that theories of metacognition are describing. To foreshadow, although there are a variety of measures of perceptual, decision, and memory uncertainty, I argue that while self-report measures are the simplest and most effective measure of certainty in causal judgments, implicit measures of uncertainty may also prove useful.

\section{Self-Report Measures}

Perhaps the simplest method of obtaining certainty reports is to ask the participant: "how certain are you in your response?" Indeed, this method is widely used in metacognitive research (Adler \& Ma, 2018; Boldt \& Yeung, 2015. De Martino et al., 2013 Fleming \& Lau, 2014; Folke et al., 2016, Li \& Ma, 2020). But there are also other related self-report measures serving various purposes. In particular, measures like judgments of learning (i.e., judgments that future recall of a memory will be successful), or judgments of the probability that a decision is correct have been used as proxies of confidence that have more directly interpretable values in terms of subjective performance (Fleming \& Dolan, 2012). Furthermore, the only widely recognized disadvantage of self-report measures is that non-human animals (e.g., mice, rats, and dogs) cannot provide responses on these measures. This poses great challenges for studying uncertainty in non-human animals, but studies of singular causal judgment focus exclusively on human participants. And even if researchers discovered a way to observe causal judgments in non-human animals, it is not clear that non-human animals can make meaningful singular causal judgments, let alone report on their uncertainty in such judgments. Altogether, self-report measures of uncertainty can be (and have already been) applied to the domain of causal judgments, and may prove as useful for research on certainty in causal judgment as they have been for research on metacognition more broadly (Liljeholm, 2015; Liljeholm \& Cheng, 2009; O'Neill et al., 2021).

\section{Opt-Out Responses}

One way of assessing certainty in both human and non-human participants is to use one of various forms of opt-out responses. In two alternative forced-choice tasks, many researchers add a pre-decision choice or a third "uncertain" choice allowing the participant to decline making a response to a particular stimulus (Kepecs \& Mainen, 2012). But in addition to doubts about the validity of such measures in non-human animals, these measures do not allow participants to give both a response and a confidence estimate in a single trial (Kepecs \& Mainen, 2012). In general, researchers do need information about both the participants' choice and their uncertainty in order 
to distinguish various models of uncertainty, making this set of measures undesirable for the study of certainty in causal judgment.

\section{Post-Decision Wagers}

Another measure of uncertainty, known as post-decision wagering, asks participants to bet on how likely their response is to be correct. Then the wagered amount is either kept or lost depending on whether the previous response was in fact correct or incorrect. It is observed that participants will make larger gambles when they are more confident (Fleming \& Dolan, 2012; Kepecs \& Mainen, 2012; Kiani \& Shadlen, 2009). At first glance, this measure is very attractive; non-human animals can be trained to wager, and the size of the wager directly reflects the extent to which a participant is willing to commit to their response. Since whether the participant wins or loses the bet depends on whether their response accurately reflects the stimulus, post-decision wagering relies on there being an objectively correct response. As I will discuss in the Problem with Causal Judgments, there is continued debate as to whether causal relations are objective phenomenon and whether people have one or more concepts of causation. In any case, it is unclear how one could demonstrate to a participant that a given causal judgment is correct or incorrect without presupposing a theory of causal judgment.

\section{Implicit Measures}

Various implicit measures, including reaction time, bodily movements, eyetracking measures, neural recordings, changes of mind, and the time a participant is willing to wait for a potential reward, also allow researchers to assess a (human or non-human animal) participant's uncertainty in a particular response (Boldt \& Yeung, 2015; Dotan et al., 2018; Folke et al., 2016; Gerstenberg et al., 2017; Kepecs et al., 2008; Ma \& Jazayeri, 2014; Stolyarova et al., 2019). Such measures have proven to be invaluable for establishing neural mechanisms for metacognition across species, for gathering information about uncertainty without interrupting a participant's behavior in a task, and for corroborating results across different methodologies (Kepecs \& Mainen, 2012). Implicit measures thus serve as a promising tactic for studying uncertainty in singular causal judgments, so long as they can be validated against self-report measures or objective accuracy to ensure that they truly reflect uncertainty, rather than some related process.

\section{Model-based Measures}

In addition to developing measures of uncertainty, researchers in metacognition have also carefully crafted measures of the degree to which a participant's uncertainty reflects the objective accuracy of their response (Fleming \& Dolan, 2012, Fleming \& Lau, 2014, Maniscalco et al., 2020; Peters \& Lau, 2015; Peters et al., 2017). Consider the case of the fad diet mentioned in the Introduction: just because one is very 
(a)

Table 1 (b)

$$
\text { Confidence }
$$

\begin{tabular}{|c|c|c|}
\hline \multirow{2}{*}{0} & 0 & 1 \\
\hline & $C R$ & $F A$ \\
\hline 1 & $M$ & $H$ \\
\hline
\end{tabular}

Classification of (a) responses by signal detection theory and (b) confidence judgments by type 2 signal detection theory. In type 1 SDT, a response is a correct rejection (CR) when the signal is absent and correctly identified as absent, a false alarm (FA) when an absent signal is mistakenly identified as present, a hit (H) when a present signal is correctly identified as present, and a miss $(M)$ when a present signal is mistakenly identified as absent. In type 2 SDT, a confidence rating is a correct rejection when the type 1 response is incorrect and unconfident, a false alarm when a response is incorrect but confident, a hit when a response is correct and confident, and a miss when a response is correct but unconfident.

confident that the diet caused weight loss does not mean that the diet was actually causally relevant for it. Though one could simply correlate confidence and accuracy, this does not account for the possibility that some participants may simply report being more confident than others, even when their amount of uncertainty is the same. The framework of signal detection theory (SDT) allows researchers to de-confound these possibilities. Type 1 SDT categorizes responses relative to the actual value of the stimulus, and distinguishes between bias - one's overall tendency to make a given response - and sensitivity - how much one's response depends on the actual value of the stimulus (see Table 1a). Similarly, type 2 SDT categorizes uncertainty judgments relative to the actual accuracy of the type 1 response, and distinguishes between metacognitive bias - one's overall tendency to be confident - and metacognitive sensitivity - how much one's confidence depends on the actual accuracy of the type 1 response (see Table 1b). Type 2 SDT also allows researchers to infer metacognitive efficiency, which is the proportion of sensory evidence that a participant employs when making confidence judgments (Fleming \& Lau, 2014 Maniscalco et al., 2016; Samaha \& Denison, 2020; Shekhar \& Rahnev, 2020). Together, these measures allow researchers to determine whether their participants' confidence ratings are appropriately calibrated to their objective accuracy. But as with post-decision wagering, I will argue in the next section that singular causal judgments may not have an objective accuracy upon which to define these measures in the usual manner.

\section{The Problem with Causal Judgments}

In this section I noted that post-decision wagers and model-based measures may not be applicable to confidence in causal judgments. Here I will provide an 
argument underlying this conclusion. To make use of post-decision wagers or modelbased measures of metacognition, a researcher needs to be able to identify (a) the participant's actual response and (b) the objectively correct response to that particular stimulus. In perception, for instance, one needs to know whether the participant detected a stimulus on a given trial, as well as whether there actually was a stimulus on that trial. In this case, determining whether a stimulus was presented on that trial is as simple as checking a digital record of whether the stimulus was presented. But we have reason to doubt that this is the case for causal judgments. I will focus on two distinct intuitions that there may not be an objectively correct causal judgment for a single occurrence of a candidate cause and an effect: the extrinsicality of causal relations and causal pluralism.

Hume (2020) famously reasoned that singular causation cannot be directly observed. That is, given only a single sequence of events $C$ and $E$, it is impossible to know whether $C$ brought about $E$, or whether $C$ just happened to precede $E$. Hume argued that what allows us to draw causal inferences is a constant conjunction of (or connection between) $C \mathrm{~s}$ and $E \mathrm{~s}$, separate from the occurrence of this $C$ and that $E$. What this implies is that causation is an extrinsic relation: whether $C$ caused $E$ doesn't just depend on $C$ and $E$ themselves, but also some regularity or dependency between $C$ s and $E$ s. While there is certainly much debate about whether causation is extrinsic in this way, most theories of causation (including counterfactual theories) do treat it as extrinsic (Beebee, 2009; Danks, 2013; Menzies, 1999). But if singular causation is an extrinsic relation that is not directly observable, then how can we determine the objectively correct causal judgment using only the single instance presented to the participant? Moreover, if we were to give a participant feedback that their causal judgment was incorrect, how could we demonstrate to the participant that their judgment was indeed incorrect on the basis of the presented stimulus alone? If causation is an extrinsic relation, then this task is impossible.

Another reason to doubt that there is a single, objectively correct, causal judgment for a given stimulus is that people may have more than one folk concept of causation (a belief known as causal pluralism, Gerstenberg et al., 2020; GodfreySmith, 2009 Hitchcock, 2007 Lombrozo, 2010, Sprenger, 2018). Causal pluralism arose out of the observation that none of our current theories of causal judgment seem to account for all of the different judgments that people make. So if people do not respond strictly according to one theory, it is possible that they sometimes respond according to one theory, and sometimes to others. So whereas most theorists would see evidence against a theory of causal judgment as disconfirming the theory in general, causal pluralists see the evidence merely as indicating that people use a different concept of causation in making those judgments, allowing for the possibility that the theory can still explain other judgments that people make. If causal pluralism is an extant (even attractive) model for causal judgment, then we cannot identify the objectively correct causal judgment for a given scenario, because what the objectively correct judgment is depends on what concept of causation participants are using. 
There is still a way in which we can make use of model-based measures, however. Perhaps if we cannot objectively determine the correct causal judgment for a given stimulus, it suffices to determine the predicted causal judgment relative to a particular theory of interest. For instance, instead of computing a participant's sensitivity to the matter of fact of whether $C$ caused $E$, we can compute the participant's sensitivity to whether the Power PC model determines that $C$ caused $E$ (Cheng, 1997). Likewise, instead of computing the relation between a participant's confidence and the objective accuracy of their causal judgments, we can compute the relation between their confidence and the correspondence between their causal judgments and the predictions of the Power PC model. This model-relative approach may prove fruitful for model-based measures of uncertainty, but it will not allow us to use post-decision wagering. If a participant makes a judgment inconsistent with a causal theory and makes a wager on their accuracy, researchers have no basis upon which to tell the participant that their judgment is incorrect without providing an independent argument that the chosen model is normative for the situation of interest. If either of the above intuitions hold, no such argument exists. So, it is unlikely that the study of certainty in causal judgments can benefit from the use of post-decision wagering.

\section{Modeling Uncertainty}

Now that I have identified several measures used in research on metacognition and argued for their relative utility in measuring uncertainty in causal judgments, I will now consider two broad classes of models of metacognitive uncertainty, with examples in each class. This list is not exhaustive, but does encapsulate most models used by researchers in metacognition. I will treat each class of models under the lens of probabilistic Bayesian inference, which is widely used in metacognition research, though these models are also compatible with a non-Bayesian interpretation (Hangya et al., 2016, Kepecs \& Mainen, 2012; Ma \& Jazayeri, 2014).

\section{Hypothesis Testing}

Under one perspective, people make decisions in the roughly the same way that statisticians do: by testing hypotheses. That is, given a two alternative forcedchoice task, people encode a noisy representation of the incoming stimulus, and they must test the hypothesis that the stimulus belongs to one of the two categories. Researchers in metacognition extended this framework to judgments of confidence, where people additionally test the hypothesis that their choice is correct (Fleming \& Daw, 2017; Hangya et al., 2016, Meyniel \& Dehaene, 2017; Meyniel et al., 2015; Peters \& Lau, 2015; Peters et al., 2017; Pouget et al., 2016). I will focus exclusively on signal detection theory (SDT), which has been influential in providing modelbased measures of metacognition and normative explanations of confidence reports (Fleming \& Dolan, 2012; Fleming \& Lau, 2014; Kiani \& Shadlen, 2009). But there are many other models, including evidence accumulation or drift-diffusion models (which generalize SDT over time), attractor models, and classifier models, which all apply 
similar principles of Bayesian decision theory to derive measures of uncertainty with largely the same patterns of confidence measures predicted by SDT and observed in human and non-human animals (Adler \& Ma, 2018, Folke et al., 2016 Kepecs \& Mainen, 2012, Kepecs et al., 2008).

Signal detection theory assumes that people encode a noisy representation $P(\hat{S}=\hat{s} \mid S=s)$ of a stimulus $S=s$. Using Bayes' rule (or some approximation), people invert this probability to determine the probability of each stimulus category given the representation: $P(S=s \mid \hat{S}=\hat{s})=\frac{P(S=s) P(\hat{S}=\hat{s} \mid S=s)}{P(\hat{S}=\hat{s})}$. To decide which response to select, people then test the hypothesis that the stimulus came from a given category $s_{1}$ or $s_{2}$ by computing a decision variable called the log posterior odds $d(s)=\log \frac{P\left(S=s_{1} \mid \hat{S}=\hat{s}\right)}{P\left(S=s_{2} \mid \hat{S}=\hat{s}\right)}=\log \frac{P\left(S=s_{1}\right)}{P\left(S=s_{2}\right)}+\log \frac{P\left(\hat{S}=\hat{s} \mid S=s_{1}\right)}{P\left(\hat{S}=\hat{s} \mid S=s_{2}\right)}$. Assuming a uniform prior distribution over $S$ between $s_{1}$ and $s_{2}, d(s)$ is just the likelihood ratio test that the representation $\hat{s}$ is more likely when $S=s_{1}$ than when $S=s_{2}$. When $d(s)>0$, there is evidence that the stimulus $S=s_{1}$, and so people will choose the response that corresponds to $s_{1}$. When $d(s)<0$, there is evidence that the stimulus $S=s_{2}$, and so people will choose the response that corresponds to $s_{2}$ (Kepecs \& Mainen, 2012).

The innovation of researchers in metacognition was to realize that $d(s)$, in addition to predicting decisions themselves, also predicts one's certainty in that decision. In particular, whereas the sign of $d(s)$ predicts one's decision, the absolute value $|d(s)|$ predicts uncertainty in that decision. Equivalently, one could also use the raw posterior probability $P(S=s \mid \hat{S}=\hat{s}$ ) of the chosen stimulus value $s$ (Kepecs \& Mainen, 2012 Kiani \& Shadlen, 2009, Li \& Ma, 2020). Recent work has shown that the posterior probability (and likewise Frequentist $p$-values) exhibit three general properties of statistical decision confidence observed in mice: (a) confidence and accuracy are positively related (b) confidence increases with increasing evidence when a choice is correct, but decreases when the choice is incorrect, and (c) confidence predicts accuracy beyond evidence discriminability (Hangya et al., 2016, Kepecs et al., 2008).

Hypothesis testing models like SDT are extensively used in metacognition research, and have been tremendously successful in explaining confidence judgments in perception, decision-making, and memory (Adler \& Ma, 2018; Fleming \& Dolan, 2012; Li \& Ma, 2020; Maniscalco et al., 2016; Peters \& Lau, 2015; Shekhar \& Rahnev, 2020). Unfortunately, these models express confidence in a categorical choice between two or more distinct responses. Though some extant models of singular causal judgment treat causal judgments as categorical in this way (Bello et al., 2018, Khemlani et al., 2014 Sartorio, 2020 Wolff, 2007), most philosophers and psychologists now treat causal judgment as a truly graded phenomenon (Cheng, 1997; Danks, 2013, 2017; Gerstenberg et al., 2020; Halpern \& Hitchcock, 2015; Icard et al., 2017; Morris et al., 2019 Pearl, 2009; Spellman, 1997; Woodward, 2003). A recent study investigating empirical distributions of causal judgments found that though most causal judgments were discrete, people did often give graded causal judgments (O'Neill et al., 2021). If 


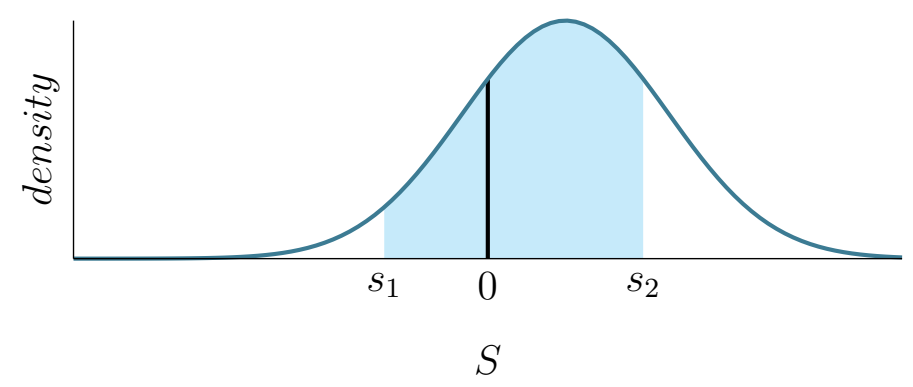

Figure 4

Example probability distribution of a continuous stimulus $S$. The y-value represents probability density, whereas the area under the curve represents probability. For a single value $S=0, P(s=0)=0$, since there is no area under a single point. But for a range of possible values $\left[s_{1}, s_{2}\right], P\left(s_{1} \leq S \leq s_{2}\right)=\int_{s_{1}}^{s_{2}} p(s) d s \geq 0$.

future research is able to explain away the existing gradation in causal judgments, then signal detection theory would provide an ideal basis for understanding uncertainty in these judgments. But provided that causal judgment is a graded phenomenon, we need a model of metacognitive uncertainty that operates on continuous responses.

\section{Parameter Estimation}

Until recently, the vast majority of research on decision-making and decision uncertainty pertained to categorical decisions that are amenable to a hypothesis testing conceptualization. But just as researchers in statistics are beginning to move beyond hypothesis testing towards methods that emphasize estimation of continuous parameters and their uncertainty (Cumming, 2014 Kruschke \& Liddell, 2018), researchers in metacognition are also generalizing their models to problems of continuous-valued choice (Meyniel \& Dehaene, 2017; Meyniel et al., 2015; Yeung \& Summerfield, 2012). To see why this difference matters, consider what the hypothesis testing framework implies for continuous values. For a categorical distribution, the posterior probability $P(S=s \mid \hat{S}=\hat{s})$ that the stimulus $S$ belongs to the category $s$ directly specifies the probability that a response of category $s$ is correct. But when $S$ is continuous, the distribution over $S$ reflects probability density, and not probabilities themselves, which are calculated as integrals over probability densities. For the distribution in Figure 4. for example, while the probability density at $S=0(p(S=0)$, indicated by the $y$-value at the black line) is non-zero, the probability that $S$ is exactly zero $P(S=0)=\int_{0}^{0} p(s) d s=0$ is zero. By contrast, the probability that $S$ lies in the range $\left[s_{1}, s_{2}\right]$ (indicated by the shaded area), $P\left(s_{1} \leq S \leq s_{2}\right)=\int_{s_{1}}^{s_{2}} p(s) d s$, is non-zero. This simple example demonstrates that unlike for categorical distributions, one cannot simply read off a probability that a continuous stimulus has precisely a single value, because that probability will always be zero.

If we cannot quantify metacognitive uncertainty in continuous stimuli as the 
probability that the stimulus has a particular value, we need to invoke a new definition of uncertainty. Thankfully, we can again borrow such a definition from Bayesian statistics: the posterior precision, or inversely, posterior width (Navajas et al., 2017; Pouget et al., 2016 Yeung \& Summerfield, 2012). The intuition behind this idea is that if the posterior distribution over possible stimulus values is narrow, then the mean will provide a precise estimate of the actual stimulus value. Conversely, if the posterior is wide, then the mean will provide an imprecise estimate of the actual stimulus value. There are a few different ways we can define posterior width. If we use the posterior mean $\mu=\mathbb{E}[s \mid \hat{S}=\hat{s}]$ (that is, the average estimated stimulus given a particular noisy representation) as an estimate of the actual value of the stimulus $s$, then it is natural to quantify the posterior width in terms of its variance $\operatorname{Var}(p(S=s \mid \hat{S}=\hat{s}))$ or its standard deviation $\sigma=\sqrt{\operatorname{Var}(p(S=s \mid \hat{S}=\hat{s}))}$ (Navajas et al., 2017). One could also use the coefficient of variation $C V(p(S=s \mid \hat{S}=\hat{s}))=\frac{\sigma}{\mu}$, which is the standard deviation of the posterior in units of the overall magnitude of the posterior. Finally, one could use the differential entropy $h(s)=-\int p(S=$ $s \mid \hat{S}=\hat{s}) \log p(S=s \mid \hat{S}=\hat{s})$, which expresses the expected amount of information or surprise conveyed by a sample from the posterior. Each of these three measures corresponds not to the probability that the estimated value of the stimulus is correct, but rather to the relative precision of the estimate (Pouget et al., 2016). Though these notions of uncertainty are qualitatively different from the notion provided by the signal detection models prevalent in metacognition research, they are ideal for describing uncertainty in the continuously-valued causal strength estimate provided by counterfactual sampling models of causal judgment.

\section{Confidence in Causal Judgments}

Now that I have identified counterfactual sampling theories of singular causal judgment and posterior precision as ideal candidates for creating a unified theory of causal metacognition, I will review the limited amount of research that has measured both participants' causal judgments and their certainty in those judgments. Though few of these studies seek to explain confidence in causal judgment, they report relations between confidence and causal judgment that are of importance for any theory of causal metacognition.

\section{Confidence in General Causal Judgments}

The bulk of the previous research on confidence in causal judgments comes from studies on general causal judgments (i.e., judgments that events of type $C$ generally cause events of type $E$ ). In such studies, participants are presented with a number of instances of some variable $E$ (e.g., the presence of a headache) before and after the occurrence of some candidate cause $C$ (e.g., the consumption of some mineral, Liljeholm \& Cheng, 2009). These data are sometimes presented all at once 
in the form of contingency tables (Liljeholm \& Cheng, 2009), and sometimes individually in succession (Collins \& Shanks, 2006 Liljeholm, 2015; Perales \& Shanks, 2003 Shanks, 1987). Based on this contingency information, the participant is asked to make a causal judgment of the sort, "To what extent does ingesting this mineral cause headaches?" In comparing these judgments with the Power PC model of causal judgment, Buehner et al. (2003) found systematic deviations from the theoretical predictions of the model. They hypothesized that these deviations could be explained, however, by assuming (a) that participants sometimes conflate the strength of a causal relation with their confidence in the relation, and (b) that confidence in causal judgments increases with the number of data points the participant uses to make the causal judgment. Liljeholm and Cheng (2009) tested this prediction, known as the conflation hypothesis, and found that participants did indeed conflate causal judgments and confidence, though these two variables remained dissociable.

Though these studies all measured and reported confidence ratings of causal judgments, they were using confidence reports in service of explaining causal judgments. But a recent line of research is directed at testing the predictions of uncertainty in causal judgments provided by the causal support model (Griffiths \& Tenenbaum, 2005 Liljeholm, 2015, 2020, Lu et al., 2008). The causal support model assumes that participants solve two distinct problem when making general causal judgments: they infer whether there exists a causal link between two variables in a causal graph (known as causal structure induction), and they estimate the strength of the causal link, assuming that it exists (known as parameter estimation, Tenenbaum \& Griffiths, 2001). Related to these two problems, there are then two kinds of uncertainty in the model. Causal support expresses uncertainty about whether there is a link between two variables in a causal graph, and is defined as the log posterior odds of the graph having a link compared to the graph having no such link. Causal support, then, is directly analogous to the log posterior odds measure used in studies of metacognition about discrete choice provided by signal detection theory (Hangya et al., 2016. Kepecs et al., 2008, Kiani \& Shadlen, 2009). By contrast, uncertainty in causal strength is expressed as the entropy of the posterior distribution over causal strength, which parallels measures of uncertainty in continuous decision-making paradigms (Liljeholm, 2015 Meyniel et al., 2015, Yeung \& Summerfield, 2012).

Liljeholm (2015) was the first effort to the causal support model's predictions of uncertainty in addition to its predictions of causal judgment. Specifically, Liljeholm (2015) looked at two potential sources of uncertainty implied by the model: confounding and interacting causes. Participants were told that four allergy medicines, $C$ (for confounded), $D, I$ (for independent), and $J$, may sometimes cause headaches as a side-effect, and it was the participant's job to determine whether each medicine caused headaches. Then they were repeatedly presented with individual cases of a patient who took no medicines, one of the four medicines, or two of the four medicines, asked to guess whether that patient developed a headache, and received corrective feedback. Finally, participants were asked to rate whether each medicine had a causal 
relationship with headaches, how strongly each medicine influenced headaches, and how uncertain they were about the causal strength rating. Critically, to test the effects of confounding and independence on uncertainty, they had participants complete this task twice, with different frequencies of the four medicines. The medicines were paired to separate the effects of confounding and independence such that $C$ and $D$ never co-occurred with $I$ and $J$, but medicines within each pair could co-occur with each other. So, I will discuss the results for the confounded causes $(C$ and $D)$ separately from the interacting causes $(I$ and $J)$.

In the first phase, $C$ and $D$ were confounded, meaning that whenever $C$ was present $D$ was also present, and whenever $C$ was absent $D$ was also absent. In the second phase, however, $C$ was allowed to occur without $D$. The causal support model predicts that uncertainty should be lower in the second phase, since the observation of $C$ without $D$ allows for the effects of $C$ and $D$ to be de-confounded. And this is what Liljeholm (2015) observed: participants were more likely to rate that they could not establish a causal relation between $C$ and headaches, they rated the causal strength of $C$ as more ambiguous, and they rated higher uncertainty in causal strength when $C$ was confounded with $D$. In contrast to the confounded causes $C$ and $D, J$ was allowed to occur in the absence of $I$, but $I$ only ever occurred with $J$ in the first phase. The causal support model predicts that participants should have relatively high certainty here, because the occurrence of $J$ without $I$ allows participants to separate the relative influences of $J$ and $I$ on headaches. In the second phase, $I$ was also allowed to occur without $J$, but the effect of $I$ on headaches in these trials suggested the effects of $I$ and $J$ on headaches were not independent. For example, if both $I$ and $J$ are needed for headaches to occur, just looking at the effect of $J$ by itself and $I$ and $J$ together would suggest that $I$ is solely the cause of headaches. But after observing that headaches aren't caused by $I$ itself, participants must reevaluate their causal model to consider that $I$ and $J$ are both needed. The causal support model predicts that there should be even more certainty about the influence of $I$ in the second phase, because viewing the effects of $I$ and $J$ in isolation allows for better separation of their relative influences. Although the causal support model accounted for the fact that causal ratings of $I$ tended to be lower in the second phase, however, its predictions were inconsistent with the finding that participants were actually less certain about their causal strength ratings in the second phase than in the first phase. Thus, while the causal support model successfully predicted decreased confidence in the existence and strength of a causal relationship when a cause's influence was confounded, it failed to account for decreased confidence when the cause's influence depended on the value of another variable (Liljeholm, 2015).

A later study employing a similar paradigm investigated whether the decrease in uncertainty due to causal confounding was reflected in BOLD activity recorded with functional magnetic resonance imaging (Liljeholm, 2020). This study found that BOLD activity in the dorsomedial prefrontal cortex, dorsolateral prefrontal cortex, and lateral superior frontal gyrus was sensitive to uncertainty in the presence of 
causal confounding, as predicted by the causal support model. Moreover, the pattern of BOLD activity predicted by the causal support model was differentiated from BOLD activity that was simply related to trial-by-trial stochasticity or error-related feedback. So although the causal support model does not account for all observed patterns in uncertainty in general causal judgments, it is fostering productive research towards this end.

\section{Confidence in Singular Causal Judgments}

Much less research is available on confidence in singular causal judgments (i.e., judgments that this particular event $C$ caused that particular event $E$ ). To my knowledge, only a single study has recorded measures of confidence in singular causal judgments. In two experiments, O'Neill et al. (2021) tested two competing (but not mutually exclusive) explanations that singular causal judgments are graded (i.e., non-binary). The first experiment tested the explanation that participants provide graded causal judgments when they are uncertain, but binary causal judgments when they are certain (similar to the reliability hypothesis for general causal judgments). Indeed, participants did tend to give more graded causal judgments when they were uncertain. The second experiment tested the explanation that people give graded causal judgments when an event is causal, but its causal influence is weak. In line with this explanation, changes in singular causal judgments due to normality, causal structure, and the number of candidate causes could not be explained by changes in confidence. O'Neill et al. (2021) interpreted these results to mean that people give graded ratings both when they are uncertain and also when the influence of a candidate cause is weak. While this study utilized confidence ratings to determine whether singular causal judgments are graded, no research to date has investigated how people come to estimate their uncertainty in a singular causal judgment. In the following section, I will provide the first attempt at such an account.

\section{A Unified Model of Causal Metacognition}

In the previous sections, I have argued that counterfactual sampling theories, due to their reliance on probabilistic inference, are the most amenable to making predictions about confidence in singular causal judgment. I have also argued that while popular hypothesis testing models of metacognition do not apply to continuous causal judgments, parameter estimation frameworks can be applied to this domain. After reviewing the limited amount of research on confidence in causal judgments, I noted that there currently exist no accounts of how people estimate their uncertainty in singular causal judgments. To provide the first unified model of certainty in causal judgment, in this section I will extend counterfactual sampling theories with parameter estimation models of metacognitive uncertainty. Though the proposed measures are normative extensions of counterfactual sampling theories (i.e., they describe how people should estimate uncertainty if counterfactual sampling theories are correct), 
more work is necessary to determine whether any of these measures are descriptively accurate (i.e., whether people actually estimate certainty in this way).

\section{Monte-Carlo Estimation}

Counterfactual sampling theories of singular causal judgment suggest that to determine the extent to which some event $C$ caused another event $E$, people imagine a number of possibilities in which $C$ did or did not happen, and they check whether $E$ would still happen in that possible world. Because people cannot imagine all such possibilities (there may be infinitely many of them), it is assumed that they only sample a small subset of these possibilities, although how precisely that subset is selected is hard to say. Finally, people estimate the causal strength of $C$ on $E$ as the average of the difference $C$ made to $E$ in all of the imagined possibilities. As I described in the section Measures of Singular Causal Strength, this process of estimating a probability through sampling can be characterized as an iterative Monte Carlo algorithm (duplicated for convenience, where the subscript $n$ refers to the sample number, $U_{n}$ are exogenous variables meaning that their causes are not modeled, $C_{n}$ and $E_{n}$ are endogenous variables meaning that their values depend only on the exogenous variables, $\mathcal{F}$ is the set of structural equations defining the

endogenous variables in terms of the exogenous variables, $E_{n_{C_{n}=c}}\left(U_{n}\right)$ is the value that $E$ would have taken in the world specified by $U_{n}$ if $C_{n}$ had the value $c, \kappa_{n, C \rightarrow E}$ is the difference $C_{n}$ made to $E_{n}$ in the world specified by $U_{n}$, and $\kappa_{C \rightarrow E}$ is the average causal influence of $C$ on $E$ ):

1. Sample a set of possible worlds by sampling all exogenous variables $U_{n}$ according to their prior probabilities $P\left(U_{n}\right)$

2. Determine whether the cause $\left(C_{n}\right)$ and effect $\left(E_{n}\right)$ occur in each world by calculating the values of all endogenous variables using the sampled values of $U_{n}$ and the structural equations provided by $\mathcal{F}$

3. Determine the specific causal effect $\kappa_{n, C \rightarrow E}$ in each world as a function of $E_{n}$ and some computed counterfactual(s) $E_{n_{C_{n}=c}}\left(U_{n}\right)$

4. Average all of the specific causal effects to obtain $\kappa_{C \rightarrow E}=\frac{1}{N} \sum \kappa_{n, C \rightarrow E}$

As I discussed above, different versions of counterfactual sampling theories commit to different specifications of $\kappa_{n, C \rightarrow E}$, the specific causal effect of $C$ on $E$. Furthermore, there is no general consensus as to which of these specifications is normatively correct, or as to which (if any) is actually used by people when making causal judgments. Below I will use the measure provided by Quillien (2020) as an example, because it has been shown to best account for observed causal judgments in a simple two-cause scenario, and because (under certain assumptions) it has a simple interpretation as the correlation coefficient between the cause and effect. To make 
things even more concrete, I will restrict predictions of certainty in causal judgments to the following vignette (Morris et al., 2019):

A person, Joe, is playing a casino game where he reaches his hand into two boxes and blindly draws a ball from each box. He wins a dollar if and only if he gets a green ball from the left box and a blue ball from the right box.

Joe closes his eyes, reaches in, and chooses a green ball from the first box and a blue ball from the second box. So Joe wins a dollar.

Please tell us much you agree or disagree with this statement: Joe's first choice (where he chose a green ball from the first box) caused him to win the dollar.

In an experiment using this vignette, participants are also told the probability that Joe will draw a green ball from the left box and the probability that Joe will draw a blue ball from the right box. Since participants are asked about Joe's choice of the green ball, I will refer to Joe's choice of the green ball as $C$ (i.e., the focal cause), Joe's choice of the blue ball as $A$ (i.e., the alternate cause), and Joe's winning a dollar as $E$. I will also refer to the probabilities of these three events as $P(C), P(A)$, and $P(E)$, respectively. The predicted judgments that drawing a green ball caused Joe to win a dollar are plotted in Figure 5 as a function of the probabilities that Joe draws a green and blue ball (Quillien, 2020). As discussed earlier, this measure is simply the correlation coefficient between Joe drawing a green ball and Joe winning a dollar in the set of imagined counterfactual worlds. I will note two important patterns in these predictions that have been observed in the literature on causal judgment. First, the model predicts abnormal inflation: people judge $C$ to be more causal when it is abnormal (i.e., when $P(C)$ is low) compared to when it is normal (i.e., when $P(C)$ is high, Icard et al., 2017). People are likely to determine that events are more causal when they are abnormal because when an event is rare, it is easy to imagine the event not having happened in the first place. In contrast, it is harder to "undo" the occurrence of events that events that are normal, because they occur in most possible worlds that one can imagine. So, even though the difference that $C$ makes to $E$ is independent of whether $C$ is normal or abnormal, people judge $C$ 's influence on $E$ to be bigger when it is rare since it is more apparent that $C$ made a difference to $E$ in this case (Icard et al., 2017; Kahneman \& Tversky, 1981). Second, the model predicts causal superseding: people judge $C$ as less causal when the alternate cause $A$ is abnormal (i.e., when $P(A)$ is low) compared to when $A$ is normal (i.e., when $P(A)$ is high, Kominsky et al., 2015). The explanation for causal superseding is similar to the explanation for abnormal inflation. When $A$ is rare, it is easy to imagine $A$ not happening in the first place. So, when people counterfactually manipulate $C$ by imaging possibilities in which $C$ did not occur, they usually also imagine that $A$ did not occur either. Since both $C$ and $A$ are required for $E, C$ does not make a difference to $E$ in the absence of $A$, and so people are likely to rate $C$ as less causal when $A$ is abnormal because they evaluate $C$ 's effect on $E$ in the absence of $A$. When $A$ is normal, in contrast, it is harder to imagine $A$ not occurring, so people evaluate the effect of $C$ on $E$ in the presence of $A$. Since $C$ does affect $E$ in the presence of $A$, 


\section{Figure 5}

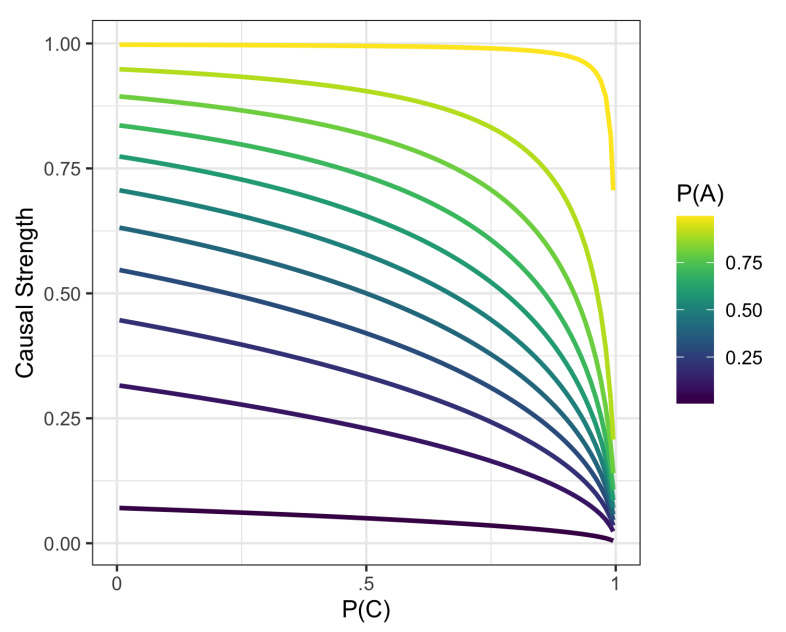

Predicted causal judgments from Quillien (2020) of $C$ on $E$ as a function of $P(C)$ and $P(A)$, where $A$ is an alternate cause.

people rate $C$ as more causal in this case. Though I use this example and model for demonstration, the proposed measures of uncertainty in causal judgments easily generalize to other causal structures and other counterfactual sampling models.

Now that I have identified counterfactual sampling models and posterior precision as viable candidates for a study of causal metacognition and identified a suitable context for representing my predictions, I will propose four different ways that people could quantify uncertainty in their estimates of causal strength. Each of these ways makes use of the fact that counterfactual sampling models estimate the strength of a cause through Monte-Carlo estimation: that is, they presume that people imagine a set of counterfactual possibilities, and they average the difference that $C$ makes to $E$ in all of those possibilities. If people imagine all of these possibilities and compute the difference that $C$ makes to $E$ in each of these possibilities to form a causal judgment, it is natural to assume that they use the same information to estimate their uncertainty in that causal judgment. That is, whereas people estimate the causal effect of $C$ on $E$ as the average of the distribution of differences that $C$ made to $E$ in all of imagined possibilities, they estimate their uncertainty as some other function over that distribution. Out of the four metrics that I propose, the first (Monte-Carlo Standard Error) is normative in the sense that if people estimate causal strength using Monte-Carlo estimation, this metric is the optimal way to quantify uncertainty in that causal strength metric. Additionally, all four metrics are normative in the weaker sense that people reuse the same information to estimate uncertainty that they use to estimate causal strength, so that no additional computations or assumptions need to be made to estimate uncertainty. 


\section{Monte-Carlo Standard Error}

When researchers calculate a sample mean of some data as an estimate of the population mean, they use the standard error (SE) as a normative measure of the uncertainty in this estimate. Since counterfactual sampling theories assume that people estimate causal strength as the average of sampled specific causal effects, the optimal way for them to estimate uncertainty in causal strength is also to use the standard error. Formally, if people estimate causal strength as the average difference $C$ makes to $E, \kappa_{C \rightarrow E}=\frac{1}{N} \sum \kappa_{n, C \rightarrow E}$, then the best measure of uncertainty over their estimate is the standard error $S E\left(\kappa_{C \rightarrow E}\right)=\sqrt{\frac{\operatorname{Var}\left(\kappa_{n, C \rightarrow E}\right)}{N}}$. The reason that this measure is normative is that assuming that people sample $N$ counterfactuals randomly, the standard error is precisely the extent to which the average causal strength estimates are expected to vary under replication. Since the number of sampled counterfactual possibilities $N$ is not known in practice, it suffices to use the standard deviation as a predictor of uncertainty. We can predict, however, that uncertainty will also vary with $\frac{1}{\sqrt{N}}$. The derived standard deviation of specific causal effects provided by Quillien (2020) is plotted in Figure 6a (see the Appendix for derivation).

\section{Variance}

Instead of using the standard deviation or standard error of the sampled specific causal effects, it is possible that people use the variance of these samples, equal to the square of the standard deviation. When the distribution of specific causal effects is known, the variance can be derived as the square of the average specific causal effect subtracted from the average squared specific causal effect (i.e., $\operatorname{Var}\left(\kappa_{n, C \rightarrow E}\right)=\mathbb{E}\left[\kappa_{n, C \rightarrow E}^{2}\right]-\mathbb{E}\left[\kappa_{n, C \rightarrow E}\right]^{2}$. The derived variance in specific causal effects predicted by Quillien (2020) is plotted in Figure 6b (see the Appendix for derivation).

\section{Coefficient of Variation}

People could also estimate their uncertainty in a singular causal judgment relative to the magnitude of the causal judgment itself. The coefficient of variation provides such a scale-free metric, and is computed as the ratio of the standard devia-

tion of specific causal effects and the estimated causal judgment, $\frac{\sqrt{\operatorname{Var}\left(\kappa_{n, C \rightarrow E}\right)}}{\kappa_{C \rightarrow E}}$. This quantity can be understood as the size of the standard deviation relative to the size of the mean. The derived coefficient of variation predicted by Quillien (2020) is plotted in Figure 6c (see the Appendix for derivation).

\section{Entropy}

Finally, people could use the entropy of the distribution of specific causal effects as a measure of uncertainty in their singular causal judgment. When the distribution of specific causal effects is discrete with possible outcomes $\kappa_{C \rightarrow E_{i}}$, the entropy is $H\left(\kappa_{C \rightarrow E}\right)=-\sum_{i} P\left(\kappa_{C \rightarrow E_{i}}\right) \log P\left(\kappa_{C \rightarrow E_{i}}\right)$. When the distribution of specific causal 
effects is continuous, the entropy is $H\left(\kappa_{C \rightarrow E}\right)=-\int p\left(\kappa_{C \rightarrow E_{i}}\right) \log p\left(\kappa_{C \rightarrow E_{i}}\right) d \kappa_{C \rightarrow E_{i}}$. In both cases, the entropy can be interpreted as the expected amount of surprise, or the expected gain in information, given an observed specific causal effect $\kappa_{n, C \rightarrow E}$. The entropy of specific causal effects predicted by Quillien (2020) is plotted in Figure $6 \mathrm{~d}$ (see the Appendix for derivation).

\section{Alternatives to Monte-Carlo Estimation}

The four proposed measures of uncertainty in causal judgment have the benefit that they are directly implied by counterfactual sampling models. But, of course, actual ratings of confidence in causal judgment may not be well-described by these measures. In anticipating this possibility, I now provide two possible ways that counterfactual sampling models could be extended to account for these possible findings, given some additional assumptions.

\section{Importance Sampling}

Until now, I have assumed that people randomly sample possible worlds according to the statistical normality of those worlds. Since $C$ and $A$ are independent, this means that the probability that people sample $C$ to occur is $P(C)$, and the probability that people sample $A$ to occur is $P(A)$. Although it has been shown that people tend to imagine possible worlds that are normal more often than those that are abnormal (Kahneman \& Miller, 1986 Kahneman \& Tversky, 1981), it has not been shown that people sample possible worlds directly in proportion to their prior probabilities. In fact, it may actually be desirable for people to over-sample possible worlds that are unlikely but informative or valuable, and recent work has provided some support for this idea (Bear et al., 2020; Phillips et al., 2019). One particular reason that this might be the case is provided by the culpable control model of causal judgments, which predicts that people determine $C$ to be a cause of $E$ when they have a negative evaluation of $C$ and they want to confirm ascriptions of blame to $C$ for $E$ (Alicke et al., 2011). Counterfactual sampling theories could potentially explain this finding by assuming that people, in search of blaming $C$ for $E$, disproportionately sample worlds in which $C$ makes a difference to $E$. To account for this possibility, we can assume that people instead sample possible worlds according to some other probability distribution $Q(U)$ over the exogenous variables $U$. Recovering an estimate of causal strength over $P$, then, involves only a simple algebraic manipulation:

$$
\begin{aligned}
\mathbb{E}_{P}\left[\kappa_{C \rightarrow E}(U)\right] & =\int \kappa_{C \rightarrow E}(U) P(U) d U \\
& =\int \kappa_{C \rightarrow E}(U) \frac{P(U)}{Q(U)} Q(U) d U \\
& =\mathbb{E}_{Q}\left[\kappa_{C \rightarrow E}(U) \frac{P(U)}{Q(U)}\right]
\end{aligned}
$$




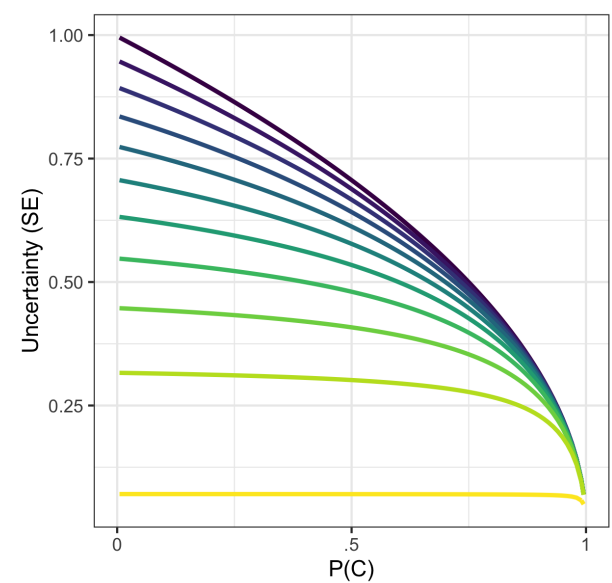

(a) Standard Error

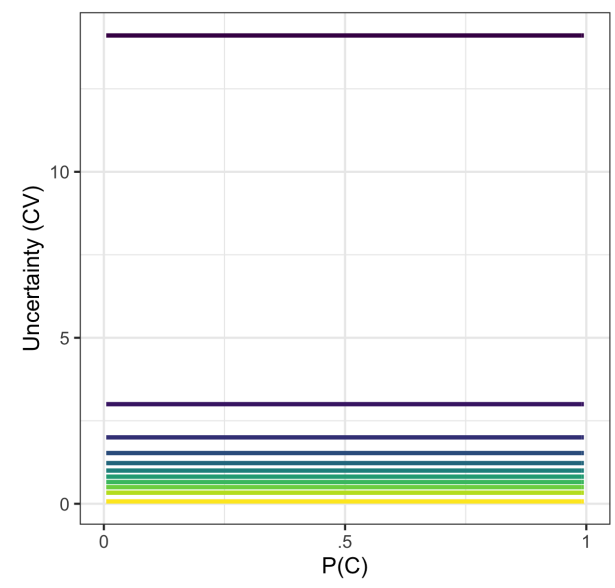

(c) Coefficient of Variation

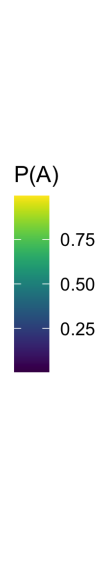

(b) Variance

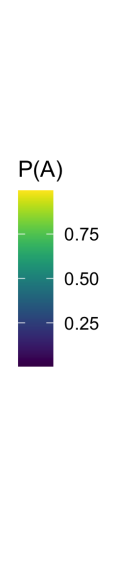

(d) Entropy
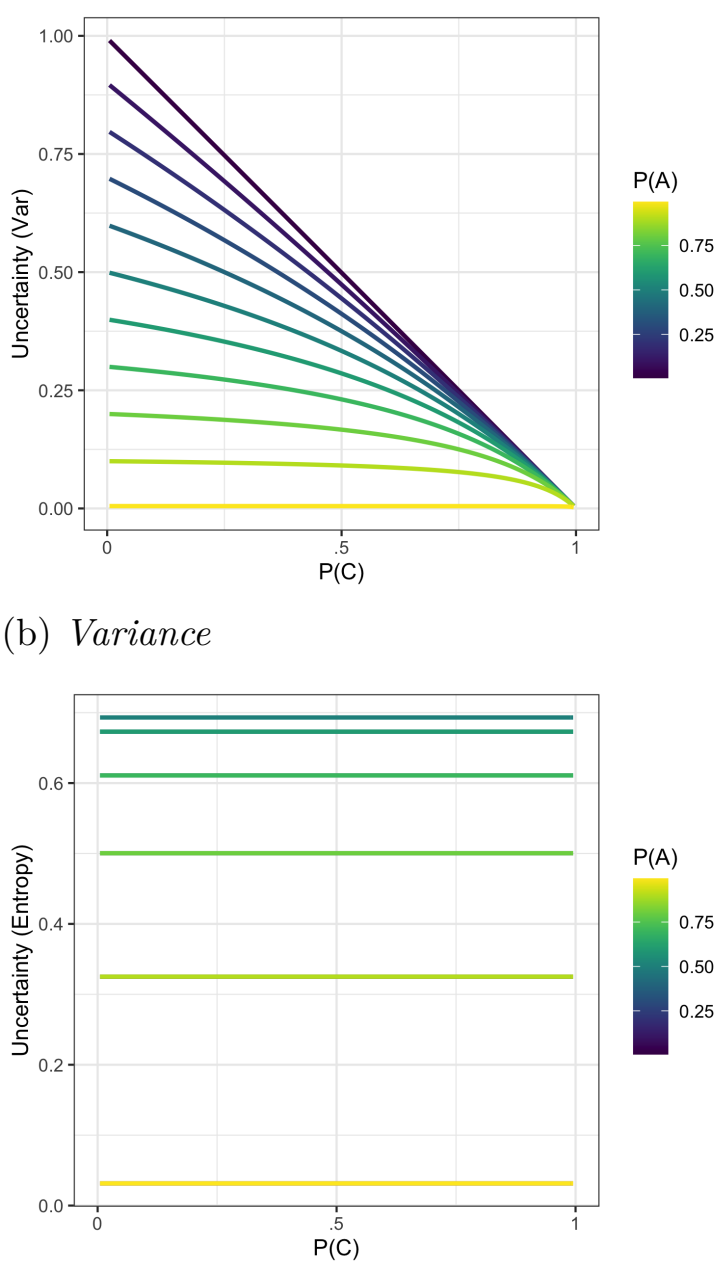

Figure 6

Predicted uncertainty in singular causal judgments from the standard error, variance, coefficient of variation, and entropy of Quillien (2020) of $C$ on $E$ as a function of $P(C)$ and $P(A)$, where $A$ is an alternate cause. 
This means that sampling from $Q(U)$ provides an unbiased estimate of the expected causal strength from $P(U)$, so long as $Q(U)$ covers $P(U)$ (i.e., it puts nonzero probability mass wherever $P(U)$ has non-zero mass) and one weights the obtained causal strength measures by the likelihood ratio $\frac{P(U)}{Q(U)}$. In statistics, this approach is called importance sampling, and is often used to estimate an expected value over $P(U)$ when it is difficult to sample directly from $P(U)$, or to minimize the variance in the estimate over $P(U)$ (Robert \& Casella, 2013).

Unfortunately, it is difficult to predict how an importance sampling scheme might affect uncertainty in the causal strength estimate. When the choice of sampling distribution $Q(U)$ is optimal, the variance of the resulting causal strength estimate will shrink asymptotically close to zero. But when the choice of $Q(U)$ is poor, the estimation variance can inflate towards infinity. If it can be determined how people select a counterfactual sampling distribution $Q(U)$, then, this importance sampling approach has the potential to capture a wide variety in confidence in singular causal judgments, as well as individual variability in confidence ratings due to individual variability in the choice of $Q(U)$.

\section{Causal Structure Induction}

Yet another possibility is that when evaluating confidence in singular causal judgments, people sometimes report their uncertainty that a causal relation exists (i.e., causal support), rather than their uncertainty in their causal strength estimate (Griffiths \& Tenenbaum, 2005). In studies of singular causal judgment, participants are usually explicitly given the causal structure of a scenario, so that they have no underlying uncertainty about the possible counterfactuals afforded by the stimulus (Henne, Niemi, et al., 2019 Icard et al., 2017; Morris et al., 2019). Additionally, confidence ratings of singular causal strength judgments have been observed to be high and largely insensitive to causal structure, number of causes, and normality, though it is not known how well these patterns generalize to other stimuli (O'Neill et al., 2021). If more precise studies fail to find any differences in confidence ratings, it is possible that participants are rating causal support, which is expected to be high in these scenarios since participants are given the causal structure a priori. If this is the case, new measures of uncertainty must be developed that differentiate between uncertainty in a causal link (which is expected to be high) and uncertainty in a causal strength estimate (which is expected to vary). So, to fairly test the predictions of counterfactual sampling models I have outlined above, it will be pertinent to assess the validity of measures of uncertainty in causal strength estimates.

\section{Discussion}

In this paper I have argued that the study of how people make causal judgments about particular events could greatly benefit from a corresponding study of how people manage uncertainty in these judgments, a topic I call causal metacognition. In 
pursuit of establishing this new branch of metacognition research, I reviewed several competing theories of singular causal judgment with the aim of determining which extant theories are most amenable to extension to the domain of causal metacognition, and I determined that probabilistic counterfactual sampling theories are ideal in fitting this role. Next, I reviewed broad conceptual frameworks that are employed in the study of metacognition relating to perception, memory, and decision-making, with the aim of selecting the framework that is most compatible with counterfactual sampling theories of causal judgment. Here I argued that since singular causal judgments are thought to be graded (O'Neill et al., 2021), a parameter estimation framework that uses posterior precision as a metric of uncertainty most readily applies to causal judgments. After reviewing the limited amount of research on confidence in causal judgments, I extended counterfactual sampling models of causal judgment with posterior precision metrics of uncertainty, and derived several possible measures for a recent, promising instance of a counterfactual sampling model (Quillien, 2020). To make these predictions, importantly, I did not need to modify the basic counterfactual sampling account of singular causal judgments. Instead, under the assumptions already made by these theories, my proposed extension can be thought of as the normative baseline upon which confidence ratings should be made. Finally, I discussed two plausible extensions of this proposal that could potentially account for a wider array of confidence ratings.

To ground a study of causal metacognition, future research should test the uncertainty predictions derived from counterfactual sampling models. In particular, Morris et al. (2019) compared a wide array of predictions from counterfactual sampling models to empirically observed causal judgments in a set of simple two-cause vignettes about events with defined probabilities. To test predictions of uncertainty derived from these models as well, this study could be replicated and extended to measure certainty in causal judgments. If confidence judgments resemble the predictions made by any of these models, then there is considerable reason to reject alternative theories of singular causal judgment in favor of counterfactual sampling models, since no other theory currently provides principled predictions of uncertainty in causal judgment. If, however, confidence ratings diverge from these predictions, the models leave room to be amended to account for such observations under additional assumptions (e.g., the use of importance sampling). Therefore, requiring that models of singular causal judgment also make predictions of uncertainty in these judgments provides a stronger test of these models that may prove pivotal in improving them and selecting among them.

Besides testing the predictions of counterfactual sampling models, there remains much room for future work in causal metacognition. First and foremost, the study of causal metacognition demands that we study causal judgment under the presence of uncertainty. But to measure reliable trends in causal judgments, most studies to date focus only on situations where there is very little room for uncertainty (e.g., Icard et al., 2017; Morris et al., 2019; O'Neill et al., 2021; Phillips et al., 2019). 
In particular, studies employing vignette stimuli often leave no room for uncertainty, as the text specifies all of the relevant information that the participant needs to make a causal judgment. One set of notable exceptions is a line of work on causal judgments about physical causation, for example videos of colliding billiard balls (Gerstenberg et al., 2014; Gerstenberg et al., 2020 Gerstenberg \& Icard, 2020; Gerstenberg et al., 2017). In these studies, there is uncertainty present due to perceptual uncertainty, uncertainty about counterfactual outcomes, and time constraints in the task. Since real-life causal judgments often involve different kinds of uncertainty, employing stimuli exhibiting uncertainty can only make our understanding of causal judgment more ecologically valid.

If counterfactual possibilities are used in making causal judgments, another line of research could investigate how people quantify and reduce uncertainty over counterfactuals more generally. Gerstenberg et al. (2017), for instance, found that participants tended to make eye movements towards counterfactual trajectories of objects when they were asked to evaluate causal responsibility. Furthermore, this tendency increased when the outcome of the counterfactual was ambiguous. The authors interpreted this result as implying that participants were seeking to resolve uncertainty in that counterfactual to make causal judgments, but critically uncertainty was never directly measured in this study. Further investigation into this domain could help reveal whether uncertainty drives this sort of causal learning by directing counterfactual simulation and intervention.

Yet another branch of research could investigate the role of uncertainty in causal explanation. I began with the example of the broken computer mouse, where the confident causal judgment of the tech employee seemed more actionable than the unconfident judgment made by the brother. Although the example is intuitive and motivating, no research has addressed this topic directly. Specifically, future work should address two questions: first, do people tend to qualify causal statements with information about certainty? Second, are people sensitive to this certainty information (i.e., do they take certain statements more seriously than uncertain ones)? Answering these basic questions is essential to warranting a larger study of causal metacognition in general.

In addition to helping researchers narrow the space of theories of singular causal judgment and explain certainty's role in causal judgment, the study of causal metacognition also shows promise to deepen our understanding of causal judgment itself. Causal judgment is thought to be essential to effectively intervening in the world, communicating with others, attributing blame and praise, and making informed predictions (Chockler \& Halpern, 2004; Hitchcock \& Knobe, 2009 Knobe \& Fraser, 2008; Lombrozo, 2007; Malle et al., 2014 Morris et al., 2019). But just like other cognitive processes, causal judgments do not always accurately reflect reality, restricting their efficacy for these purposes. By seeking an account of how people determine their certainty in causal judgments and how they calibrate their certainty to the utility of their causal judgments for each of these functions, we can begin to develop an expla- 
nation of why and when causal judgments sometimes fail. Further, we can begin to develop interventions to help people maintain valid causal models and avoid tempting fallacies. In the domain of causal explanation, the study of causal metacognition may contribute to our understanding of how people qualify causal explanations by their uncertainty and how people weigh competing competing explanations by their uncertainty. Causal metacognition also has implications in machine learning, where algorithms making causal inferences can benefit from being able to keep track of the amount of evidence for causal claims and adjust subsequent plans and actions according to this uncertainty. Overall, since all the benefits attributed to people's ability for causal reasoning depend on their ability to manage uncertainty, studying causal metacognition is central to developing an understanding of causal reasoning itself. 


\section{References}

Adler, W. T., \& Ma, W. J. (2018). Comparing bayesian and non-bayesian accounts of human confidence reports. PLoS computational biology, 14 (11), e1006572.

Alicke, M. D., \& Rose, D. (2012). Culpable control and causal deviance. Social and Personality Psychology Compass, 6(10), 723-735.

Alicke, M. D., Rose, D., \& Bloom, D. (2011). Causation, norm violation, and culpable control. The Journal of Philosophy, 108(12), 670-696.

Bear, A., Bensinger, S., Jara-Ettinger, J., Knobe, J., \& Cushman, F. (2020). What comes to mind? Cognition, 194, 104057.

Beebee, H. (2009). Causation and observation.

Bello, P., Lovett, A. M., Briggs, G., \& O'Neill, K. (2018). An attention-driven computational model of human causal reasoning. CogSci.

Bernstein, S. (2014). Omissions as possibilities. Philosophical Studies, 167(1), 1-23.

Boldt, A., \& Yeung, N. (2015). Shared neural markers of decision confidence and error detection. Journal of Neuroscience, 35(8), 3478-3484.

Buehner, M. J., Cheng, P. W., \& Clifford, D. (2003). From covariation to causation: A test of the assumption of causal power. Journal of experimental psychology: learning, memory, and cognition, 29(6), 1119.

Byrne, R. M. (2016). Counterfactual thought. Annual review of psychology, 67, 135157.

Cheng, P. W. (1997). From covariation to causation: A causal power theory. Psychological review, 104 (2), 367.

Cheng, P. W., \& Novick, L. R. (1990). A probabilistic contrast model of causal induction. Journal of personality and social psychology, 58(4), 545.

Chickering, M. (1996). Learning bayesian networks is np-complete. Learning from data (pp. 121-130). Springer.

Chickering, M., Heckerman, D., \& Meek, C. (2004). Large-sample learning of bayesian networks is np-hard. Journal of Machine Learning Research, 5.

Chockler, H., \& Halpern, J. Y. (2004). Responsibility and blame: A structural-model approach. Journal of Artificial Intelligence Research, 22, 93-115.

Collins, D. J., \& Shanks, D. R. (2006). Short article: Conformity to the power pc theory of causal induction depends on the type of probe question. Quarterly Journal of Experimental Psychology, 59(2), 225-232.

Cumming, G. (2014). The new statistics: Why and how. Psychological science, 25(1), $7-29$.

Danks, D. (2013). Functions and cognitive bases for the concept of actual causation. Erkenntnis, 78(1), 111-128.

Danks, D. (2017). Singular causation. The oxford handbook of causal reasoning, 201215.

Dasgupta, I., Wang, J., Chiappa, S., Mitrovic, J., Ortega, P., Raposo, D., Hughes, E., Battaglia, P., Botvinick, M., \& Kurth-Nelson, Z. (2019). Causal reasoning from meta-reinforcement learning. arXiv preprint arXiv:1901.08162. 
Davis, Z. J., \& Rehder, B. (2020). A process model of causal reasoning. Cognitive Science, $44(5)$, e12839.

De Brigard, F., Henne, P., \& Stanley, M. L. (2021). Perceived similarity of imagined possible worlds affects judgments of counterfactual plausibility. Cognition, 209, 104574.

De Martino, B., Fleming, S. M., Garrett, N., \& Dolan, R. J. (2013). Confidence in value-based choice. Nature neuroscience, 16(1), 105-110.

Dotan, D., Meyniel, F., \& Dehaene, S. (2018). On-line confidence monitoring during decision making. Cognition, 171, 112-121.

Dowe, P. (2009). Causal process theories. The oxford handbook of causation.

Fleming, S. M., \& Daw, N. D. (2017). Self-evaluation of decision-making: A general bayesian framework for metacognitive computation. Psychological review, $124(1), 91$.

Fleming, S. M., \& Dolan, R. J. (2012). The neural basis of metacognitive ability. Philosophical Transactions of the Royal Society B: Biological Sciences, 367(1594), $1338-1349$.

Fleming, S. M., \& Lau, H. C. (2014). How to measure metacognition. Frontiers in Human Neuroscience, 8, 443. https://doi.org/10.3389/fnhum.2014.00443

Folke, T., Jacobsen, C., Fleming, S. M., \& De Martino, B. (2016). Explicit representation of confidence informs future value-based decisions. Nature Human Behaviour, 1(1), 1-8.

Gershman, S. J. (2017). Reinforcement learning and causal models. The Oxford handbook of causal reasoning, 295.

Gershman, S. J., Norman, K. A., \& Niv, Y. (2015). Discovering latent causes in reinforcement learning. Current Opinion in Behavioral Sciences, 5, 43-50.

Gerstenberg, T., Goodman, N., Lagnado, D., \& Tenenbaum, J. (2014). From counterfactual simulation to causal judgment. Proceedings of the annual meeting of the cognitive science society, 36(36).

Gerstenberg, T., Goodman, N., Lagnado, D., \& Tenenbaum, J. (2020). A counterfactual simulation model of causal judgment.

Gerstenberg, T., \& Icard, T. (2020). Expectations affect physical causation judgments. Journal of Experimental Psychology: General, $149(3), 599$.

Gerstenberg, T., Peterson, M. F., Goodman, N. D., Lagnado, D. A., \& Tenenbaum, J. B. (2017). Eye-tracking causality [PMID: 29039251]. Psychological Science, 28(12), 1731-1744. https://doi.org/10.1177/0956797617713053

Godfrey-Smith, P. (2009). Causal pluralism. The Oxford handbook of causation, 326337.

Goldvarg, E., \& Johnson-Laird, P. (2001). Naive causality: A mental model theory of causal meaning and reasoning. Cognitive Science, 25(4), 565-610. https: //doi.org/https://doi.org/10.1207/s15516709cog2504\_3 
Gopnik, A., Glymour, C., Sobel, D. M., Schulz, L. E., Kushnir, T., \& Danks, D. (2004). A theory of causal learning in children: Causal maps and bayes nets. Psychological review, 111(1), 3.

Griffiths, T. L., \& Tenenbaum, J. B. (2005). Structure and strength in causal induction. Cognitive psychology, 51(4), 334-384.

Hall, N. (2007). Structural equations and causation. Philosophical Studies, 132(1), 109-136.

Halpern, J. Y., \& Hitchcock, C. (2015). Graded causation and defaults. The British Journal for the Philosophy of Science, 66(2), 413-457.

Hangya, B., Sanders, J. I., \& Kepecs, A. (2016). A mathematical framework for statistical decision confidence. Neural Computation, 28 (9), 1840-1858.

Hart, H. L. A., \& Honoré, T. (1985). Causation in the law. OUP Oxford.

Henne, P., Bello, P., Khemlani, S., \& De Brigard, F. (2019). Norms and the meaning of omissive enabling conditions.

Henne, P., Niemi, L., Pinillos, Á., De Brigard, F., \& Knobe, J. (2019). A counterfactual explanation for the action effect in causal judgment. Cognition, 190, 157-164.

Henne, P., O'Neill, K., Bello, P., Khemlani, S., \& De Brigard, F. (2021). Norms affect prospective causal judgments. Cognitive Science, 45(1), e12931.

Henne, P., Pinillos, Á., \& De Brigard, F. (2017). Cause by omission and norm: Not watering plants. Australasian Journal of Philosophy, 95(2), 270-283.

Hitchcock, C. (2007). Three concepts of causation. Philosophy Compass, 2(3), 508516.

Hitchcock, C. (2012). Portable causal dependence: A tale of consilience. Philosophy of Science, 79 (5), 942-951.

Hitchcock, C., \& Knobe, J. (2009). Cause and norm. The Journal of Philosophy, $106(11), 587-612$.

Holyoak, K. J., \& Cheng, P. W. (2011). Causal learning and inference as a rational process: The new synthesis. Annual review of psychology, 62, 135-163.

Hume, D. (2020). An enquiry concerning human understanding: A critical edition (Vol. 3). Oxford University Press. (Original work published 1748).

Icard, T. F., Kominsky, J. F., \& Knobe, J. (2017). Normality and actual causal strength. Cognition, 161, 80-93.

Jenkins, H. M., \& Ward, W. C. (1965). Judgment of contingency between responses and outcomes. Psychological monographs: General and applied, 79(1), 1.

Johnson-Laird, P., \& Khemlani, S. (2017). Mental models and causation. Oxford handbook of causal reasoning, 1-42.

Kahneman, D., \& Miller, D. T. (1986). Norm theory: Comparing reality to its alternatives. Psychological review, 93(2), 136.

Kahneman, D., \& Tversky, A. (1981). The simulation heuristic. (tech. rep.). Stanford Univ Ca Dept Of Psychology. 
Kaiserman, A. (2018). 'More of a cause': Recent work on degrees of causation and responsibility. Philosophy Compass, 13(7), e12498.

Kepecs, A., \& Mainen, Z. F. (2012). A computational framework for the study of confidence in humans and animals. Philosophical Transactions of the Royal Society B: Biological Sciences, 367(1594), 1322-1337.

Kepecs, A., Uchida, N., Zariwala, H. A., \& Mainen, Z. F. (2008). Neural correlates, computation and behavioural impact of decision confidence. Nature, 455(7210), 227-231.

Khemlani, S., Barbey, A. K., \& Johnson-Laird, P. N. (2014). Causal reasoning with mental models. Frontiers in human neuroscience, 8, 849.

Khemlani, S., Wasylyshyn, C., Briggs, G., \& Bello, P. (2018). Mental models and omissive causation. Memory \& cognition, 46 (8), 1344-1359.

Kiani, R., \& Shadlen, M. N. (2009). Representation of confidence associated with a decision by neurons in the parietal cortex. science, 324(5928), 759-764.

Kirfel, L., \& Lagnado, D. A. (2019). I know what you did last summer (and how often). epistemic states and statistical normality in causal judgements. CogSci, 575581.

Knobe, J., \& Fraser, B. (2008). Causal judgment and moral judgment: Two experiments. Moral psychology, 2, 441-8.

Kominsky, J. F., \& Phillips, J. (2019). Immoral professors and malfunctioning tools: Counterfactual relevance accounts explain the effect of norm violations on causal selection. Cognitive science, 43(11), e12792.

Kominsky, J. F., Phillips, J., Gerstenberg, T., Lagnado, D., \& Knobe, J. (2015). Causal superseding. Cognition, 137, 196-209.

Kruschke, J. K., \& Liddell, T. M. (2018). The bayesian new statistics: Hypothesis testing, estimation, meta-analysis, and power analysis from a bayesian perspective. Psychonomic Bulletin \& Review, 25(1), 178-206.

Kushnir, T., \& Gopnik, A. (2005). Young children infer causal strength from probabilities and interventions. Psychological science, 16(9), 678-683.

Lagnado, D. A., \& Sloman, S. (2004). The advantage of timely intervention. Journal of Experimental Psychology: Learning, Memory, and Cognition, 30(4), 856.

Lewis, D. (1974). Causation. The journal of philosophy, 70(17), 556-567.

Lewis, D. (1979). Counterfactual dependence and time's arrow. Nô̂s, 455-476.

Lewis, D. (2000). Causation as influence. The Journal of Philosophy, 97(4), 182-197.

Li, H.-H., \& Ma, W. J. (2020). Confidence reports in decision-making with multiple alternatives violate the bayesian confidence hypothesis. Nature communications, $11(1), 1-11$.

Liljeholm, M. (2015). How multiple causes combine: Independence constraints on causal inference. Frontiers in psychology, 6, 1135.

Liljeholm, M. (2020). Neural correlates of causal confounding. Journal of cognitive neuroscience, 32(2), 301-314. 
Liljeholm, M., \& Cheng, P. W. (2009). The influence of virtual sample size on confidence and causal-strength judgments. Journal of Experimental Psychology: Learning, Memory, and Cognition, 35(1), 157.

Lombrozo, T. (2007). Simplicity and probability in causal explanation. Cognitive psychology, 55(3), 232-257.

Lombrozo, T. (2010). Causal-explanatory pluralism: How intentions, functions, and mechanisms influence causal ascriptions. Cognitive psychology, 61 (4), 303-332.

Lombrozo, T., \& Vasilyeva, N. (2017). Causal explanation. Oxford handbook of causal reasoning, 415-432.

Lu, H., Yuille, A. L., Liljeholm, M., Cheng, P. W., \& Holyoak, K. J. (2008). Bayesian generic priors for causal learning. Psychological review, 115(4), 955.

Ma, W. J., \& Jazayeri, M. (2014). Neural coding of uncertainty and probability. Annual review of neuroscience, 37, 205-220.

Mackie, J. L. (1965). Causes and conditions. American philosophical quarterly, 2(4), 245-264.

Malle, B. F., Guglielmo, S., \& Monroe, A. E. (2014). A theory of blame. Psychological Inquiry, 25(2), 147-186.

Maniscalco, B., Castaneda, O. G., Odegaard, B., Morales, J., Rajananda, S., \& Peters, M. A. (2020). The metaperceptual function: Exploring dissociations between confidence and task performance with type 2 psychometric curves.

Maniscalco, B., Peters, M. A., \& Lau, H. (2016). Heuristic use of perceptual evidence leads to dissociation between performance and metacognitive sensitivity. Attention, Perception, \&3 Psychophysics, 78(3), 923-937.

Meder, B., Mayrhofer, R., \& Waldmann, M. R. (2014). Structure induction in diagnostic causal reasoning. Psychological Review, 121(3), 277.

Menzies, P. (1999). Intrinsic versus extrinsic conceptions of causation. Causation and laws of nature (pp. 313-329). Springer.

Menzies, P., \& Beebee, H. (2020). Counterfactual Theories of Causation. In E. N. Zalta (Ed.), The Stanford encyclopedia of philosophy (Winter 2020). Metaphysics Research Lab, Stanford University.

Meyniel, F., \& Dehaene, S. (2017). Brain networks for confidence weighting and hierarchical inference during probabilistic learning. Proceedings of the National Academy of Sciences, 114(19), E3859-E3868. https://doi.org/10.1073/pnas. 1615773114

Meyniel, F., Schlunegger, D., \& Dehaene, S. (2015). The sense of confidence during probabilistic learning: A normative account. PLOS Computational Biology, 11(6), 1-25. https://doi.org/10.1371/journal.pcbi.1004305

Morris, A., Phillips, J., Gerstenberg, T., \& Cushman, F. (2019). Quantitative causal selection patterns in token causation. PloS one, 14(8), e0219704.

Morris, A., Phillips, J. S., Icard, T., Knobe, J., Gerstenberg, T., \& Cushman, F. (2018). Causal judgments approximate the effectiveness of future interventions. 
Navajas, J., Hindocha, C., Foda, H., Keramati, M., Latham, P. E., \& Bahrami, B. (2017). The idiosyncratic nature of confidence. Nature human behaviour, 1(11), 810-818.

O’Neill, K., Henne, P., Bello, P., Pearson, J., \& De Brigard, F. (2021). Degrading causation.

Paul, L. A., Hall, N., \& Hall, E. J. (2013). Causation: A user's guide. Oxford University Press.

Pearl, J. (2009). Causality. Cambridge university press.

Pearl, J. (2019). The seven tools of causal inference, with reflections on machine learning. Communications of the ACM, 62(3), 54-60.

Pearl, J., Glymour, M., \& Jewell, N. P. (2016). Causal inference in statistics: A primer. John Wiley \& Sons.

Perales, J. C., \& Shanks, D. R. (2003). Normative and descriptive accounts of the influence of power and contingency on causal judgement. The Quarterly Journal of Experimental Psychology Section A, 56(6), 977-1007.

Peters, M. A., \& Lau, H. (2015). Human observers have optimal introspective access to perceptual processes even for visually masked stimuli. Elife, 4, e09651.

Peters, M. A., Thesen, T., Ko, Y. D., Maniscalco, B., Carlson, C., Davidson, M., Doyle, W., Kuzniecky, R., Devinsky, O., Halgren, E., et al. (2017). Perceptual confidence neglects decision-incongruent evidence in the brain. Nature human behaviour, 1(7), 1-8.

Phillips, J., Morris, A., \& Cushman, F. (2019). How we know what not to think. Trends in cognitive sciences, 23(12), 1026-1040.

Pouget, A., Drugowitsch, J., \& Kepecs, A. (2016). Confidence and certainty: Distinct probabilistic quantities for different goals. Nature neuroscience, 19(3), 366.

Quillien, T. (2020). When do we think that x caused y? Cognition, 205, 104410.

Rescorla, R. A. (1968). Probability of shock in the presence and absence of cs in fear conditioning. Journal of comparative and physiological psychology, 66(1), 1.

Robert, C., \& Casella, G. (2013). Monte carlo statistical methods. Springer Science \& Business Media.

Salmon, W. C. (1994). Causality without counterfactuals. Philosophy of Science, 61(2), 297-312.

Samaha, J., \& Denison, R. (2020). The positive evidence bias in perceptual confidence is not post-decisional. bioRxiv.

Sartorio, C. (2020). More of a cause? Journal of Applied Philosophy, 37(3), 346-363.

Shanks, D. R. (1987). Acquisition functions in contingency judgment. Learning and Motivation, 18(2), 147-166.

Shekhar, M., \& Rahnev, D. (2020). Sources of metacognitive inefficiency. Trends in Cognitive Sciences.

Sobel, D. M., \& Kushnir, T. (2006). The importance of decision making in causal learning from interventions. Memory $\& 3$ Cognition, 34 (2), 411-419. 
Spellman, B. A. (1997). Crediting causality. Journal of Experimental Psychology: General, $126(4), 323$.

Spirtes, P. (2010). Introduction to causal inference. Journal of Machine Learning Research, $11(5)$.

Sprenger, J. (2018). Foundations of a probabilistic theory of causal strength. Philosophical Review, 127(3), 371-398.

Stolyarova, A., Rakhshan, M., Hart, E. E., O’Dell, T. J., Peters, M., Lau, H., Soltani, A., \& Izquierdo, A. (2019). Contributions of anterior cingulate cortex and basolateral amygdala to decision confidence and learning under uncertainty. Nature communications, 10(1), 1-14.

Sytsma, J. (2019). Structure and norms: Investigating the pattern of effects for causal attributions.

Tenenbaum, J. B., \& Griffiths, T. L. (2001). Structure learning in human causal induction. Advances in neural information processing systems, 59-65.

Wolff, P. (2007). Representing causation. Journal of experimental psychology: General, $136(1), 82$.

Wolff, P., Barbey, A. K., \& Hausknecht, M. (2010). For want of a nail: How absences cause events. Journal of Experimental Psychology: General, 139(2), 191.

Woodward, J. (2003). Making things happen: A theory of causal explanation. Oxford university press.

Wright, R. W. (1987). Causation, responsibility, risk, probability, naked statistics, and proof: Pruning the bramble bush by clarifying the concepts. Iowa L. Rev., 73, 1001.

Yeung, N., \& Summerfield, C. (2012). Metacognition in human decision-making: Confidence and error monitoring. Philosophical Transactions of the Royal Society B: Biological Sciences, 367(1594), 1310-1321. 


\section{Appendix \\ Derivation of Uncertainty in Causal Strength}

In the example used above, the no-confounding assumption holds, and so the causal strength metric provided by Quillien $(2020)$ is simply the correlation coefficient between $C$ and $E$ in the sampled possible worlds. That is,

$$
\begin{aligned}
\kappa_{C \rightarrow E} & =\mathbb{E}\left[\kappa_{n, C \rightarrow E}\right] \\
& =r_{C, E} \\
& =\frac{\sigma_{C}}{\sigma_{E}} b_{C, E} \\
& =\frac{\sigma_{C}}{\sigma_{E}}(P(E \mid d o(C))-P(E \mid d o(\neg C))) \\
& =\frac{\sigma_{C}}{\sigma_{E}}(P(E \mid C)-P(E \mid \neg C)) \\
& =\frac{\sigma_{C}}{\sigma_{E}} P(A) \\
& =\sqrt{\frac{P(C)(1-P(C))}{P(E)(1-P(E))} P(A)} \\
& =\sqrt{\frac{P(C)(1-P(C))}{P(C) P(A)(1-P(C) P(A))}} P(A) \\
& =\sqrt{\frac{P(A)(1-P(C))}{1-P(C) P(A)}}
\end{aligned}
$$

The first step defines the correlation coefficient as the standardized regression coefficient. The second step holds through the definition of the regression coefficient, and the third through the no-confounding assumption. The fourth step holds since $E$ only occurs in the presence of both $C$ and $A$ (i.e., $E=\min (C, A)$ ), $C$ can only make a difference to $E$ in the presence of $A$. The fifth step expands the standard deviation of Bernoulli variables $C$ and $E$. The sixth step rewrites $P(E)=P(C) P(A)$, since $E=\min (C, A)$. Finally, the last step simplifies the equation by cancelling out terms (Quillien, 2020).

First, I will derive $\operatorname{Var}\left(\kappa_{C \rightarrow E}\right)$, since it will be used to derive other measures of uncertainty. Because $\operatorname{Var}(a X)=a^{2} \operatorname{Var}(X)$ for any random variable $X$ and any constant $a$, and because the variance of a Bernoulli-distributed variable $A$ is $\operatorname{Var}(A)=$ 
$P(A)(1-P(A))$, we have:

$$
\begin{aligned}
\operatorname{Var}\left(\kappa_{C \rightarrow E}\right) & =\operatorname{Var}\left(\frac{\sigma_{C}}{\sigma_{E}} A\right) \\
& =\frac{\sigma_{C}^{2}}{\sigma_{E}^{2}} \operatorname{Var}(A) \\
& =\frac{\sigma_{C}^{2}}{\sigma_{E}^{2}} P(A)(1-P(A)) \\
& =\frac{P(C)(1-P(C))}{P(C) P(A)(1-P(C) P(A))} P(A)(1-P(A)) \\
& =\frac{1-P(C)-P(A)+P(C) P(A)}{1-P(C) P(A)}
\end{aligned}
$$

From here, the standard deviation and standard error are easy to compute:

$$
\begin{aligned}
\sigma_{\kappa_{C \rightarrow E}} & =\sqrt{\operatorname{Var}\left(\kappa_{C \rightarrow E}\right)} \\
& =\sqrt{\frac{1-P(C)-P(A)+P(C) P(A)}{1-P(C) P(A)}} \\
S E\left(\kappa_{C \rightarrow E}\right) & =\sqrt{\frac{\operatorname{Var}\left(\kappa_{C \rightarrow E}\right)}{N}} \\
& =\sqrt{\frac{1-P(C)-P(A)+P(C) P(A)}{N(1-P(C) P(A))}}
\end{aligned}
$$

The coefficient of variation is the ratio between the standard deviation and the mean of specific causal effects, so it is also relatively easy to compute:

$$
\begin{aligned}
C V\left(\kappa_{C \rightarrow E}\right) & =\frac{\sigma_{\kappa_{C \rightarrow E}}}{\kappa_{C \rightarrow E}} \\
& =\sqrt{\frac{\frac{1-P(C)-P(A)+P(C) P(A)}{1-P(C) P(A)}}{\frac{P(A)(1-P(C))}{1-P(C) P(A)}}} \\
& =\sqrt{\frac{1-P(C)-P(A)+P(C) P(A)}{P(A)(1-P(C))}} \\
& =\sqrt{\frac{1-P(A)}{P(A)}}
\end{aligned}
$$


Finally, since $C$ only makes a difference to $E$ when $A$ is present, $\kappa_{C \rightarrow E}$ only has two possible values, and so the entropy of the specific causal effects is:

$$
\begin{aligned}
H\left(\kappa_{C \rightarrow E}\right) & =-\sum P\left(\kappa_{C \rightarrow E_{i}}\right) \log P\left(\kappa_{C \rightarrow E_{i}}\right. \\
& =-P(A) \log (P(A))-(1-P(A)) \log (1-P(A))
\end{aligned}
$$

